\title{
Entwicklungsphasen und -tendenzen des Kapitalismus in Westdeutsch- land (1. Teil)
}

\author{
Elmar Alvater, Jürgen Hofifmanin, Wolfgang Schöller, Willi Semmier
}

\section{Vorbemerkung}

Es geht uns im folgenden Aufsatz um die Darstellung einiger grundlegender Entwicklungstendenzen des westdeutschen Kapitalismus. Dabei wird es kaum möglich sein, einen Grad der Ausführlichkeit in der Darstellung zu erreichen, der es möglich machen würde, auf die vielen konkreten Fragen, wie sie im Zusammenhang der Klassenbewegung tagtäglich sich ergeben, eine Antwort zu geben. Aber die Klärung der Frage, welches die Entwicklungsbedingungen und -tendenzen des westdeutschen Kapitals in den nächsten Jahren sein werden, ist eine Voraussetzung für die Untersuchung und Beantwortung vieler konkreter Probleme, sei es der Einschätzung des Staates, der Gewerkschaften, der Entstehung und Dynamik von politischen Konflikten, der Entwicklungstendenzen im Ausbildungssektor usw. Dies ist der Grund, weshalb wir die nachfolgenden Ergebnisse vorlegen, obwohl sie in vielen Punkten noch bruchstückhaft sind, der weiteren methodischen Reflexion bedürfen und nur unzulänglich die Erfahrungen der Arbeiterbewegung in Westdeutschland nach dem zweiten Weltkrieg aufarbeiten und einbeziehen. Gerade der letzte Punkt scheint uns ein echter Mangel zu sein, da wir davon ausgehen, daß politische Taktik nicht einfach theoretisch abgeieitet werden kann, sondern gerade die historischen Erfahrungen der Arbeiterbewegung dabei zu berücksichtigen wären.

Wir untersuchen hier die Entwicklungstendenzen des Kapitalverhältnisses in Westdeutschland. In kapitalistischen Gesellschaften ist das Kapital das Subjekt der Bewegung dieser Gesellschaften. Aber das Kapital ist kein Ding, keine bloß quantitative Größe, auch wenn dies so erscheint und im vuigären Bewuß tsein so vorgestell1 wird. Das Kapital ist ein gesellschaf tliches Verhältnis und umschließt als solches den Widerspruch von Lohnarbeit und Kapital. Die Entwicklungstendenzen des Kapitalverhältnisses sind folglich nicht bloß als Akkumulationstendenzen des Kapitals, sondern auch als Tendenzen der Klassenkämpfe zu untersuchen. Also: Nicht nur die Bewegung der Kapitalakkumulation, sondern auch die Bewegungen der Klassenkämpfe konstituieren die Entwicklungstendenzen des Kapitalverhältnisses. So können die Klassenkämpfe nicht losgelöst von den Akkumulationsbedingungen des Kapitals und diese nicht unabhängig von den Klassenkämpfen analysiert werden.

Wir haben bei der Untersuchung der Entwicklung des westdeutschen Kapitalismus vier Hauptaspekte in den Vordergrund unserer Betrach tung gestellt:

Erstens schien es uns wichtig, den Wertbildungsprozeß unter Einbeziehung des Weltmarktzusammenhangs zu betrachten und die Bedeutung der jeweilig herrschenden technischen Durchschnittsbedingungen und der Kontinuität des Reproduktionsprozesses (angesichts der Weltmarktverflechtung) für die Größe des Wertprodukts (bzw. der Wertmassen) herauszustellen. 
Zweitens werden wir in diesem Artikel versuchen, für Wertbegriffe analoge Marktpreisbegriffe auf der empirisch - statistischen Oberfläche zu finden, um aus der Bewegung dieser empirischen Größen umgekehrt Schlïsse auf die Wertbewegung ziehen zu können.

Drittens ist es uns auf dieser empirischen Basis daher möglich, für die Bundesrepublik nachzuweisen, daß der Fall der Profitrate zumindest für den Zeitraum von 1960 bis 1970 aus den statistischen Daten zu belegen ist (während aufgrund der empirischen Daten, die sich auf die 50 er Jahre bezogen, marxistische wie bürgerliche Wissenschaftler dieses allgemeine Gesetz glaubten, widerlegen zu können). Dabei geht es uns allerdings - gerade im Gegensatz zu neueren Untersuchungen - nicht nur darum, das Verhälinis von Lohn und Profit zu analysieren, sondern um die Darstellung der wesentichen Komponenten der Profitrate und deren Bewegung.

Viertens sollen durch dieses Vorgehen die Bedingungen, unter denen die Lohnarbeiter sich reproduzieren und unter denen sie die täglichen Kämpfe mit dem Kapital austragen, herausge arbeitet werden.

Wir werden im folgenden zunächst einige methodische Probleme anreißen und dabei versuchen, die wichtigsten Faktoren von Wertbildung, Kapitalverwertung und Wachstum des Kapitals herauszuarbeiten. Dazu bedurfte es allerdings einer grundsätzlichen Klärung der verschiedenen Ebenen der Untersuchung und der Möglichkeit der Verwendung von Indikatoren, die der bürgerlichen Statistik en tnommen werden. Zum Zweck der Untersuchung der historischen Entwicklung des Kapitals werden wir dann einige Überlegungen zur Analyse von Wertbildungsprozeß und AkkumulationsprozeB des Kapitals systematisieren und sie in eine Form bringen, die es uns im weiteren erlauben wird, die in der bürgerlichen Statistik vorgefundenen Größen zumindest in ihrer Veränderung in der Zeit benutzen und daraus Schlüsse in bezug auf die inneren Tendenzen in der Entwicklung des Kapitals ziehen zu können. Dabei kommt es uns darauf an, die Entwicklung des.Ausbeutungsgrads der Arbeit, der Profite und der jeweils vorzuschießenden Kapitale als Komponenten der Bestimmung des Verwertungsgrads des Kapitals gesamtgesellschaftlich zu betrachten. Im weiteren werden wir die Zusammenhänge von Wertbildungsprozeß und Kapitalverwertung mit der Entwicklung des Weltmarkts konkreter fassen, um daraus Ansatzpunkte für die Analyse der Stellung eines nationalen Kapitals auf dem Weltmarkt und deren Bedeutung für den inländischen Reproduktions- und Wachstumsprozeß herauszuarbeiten.

Im zweiten Abschnitt des Artikels der in PROKLA 14/15 erscheinen wird, werden wir zur Untersuchung der zuvor benannten Bedinguigen des Wertbildungsprozesses und der Verwertung des Kapitals in Westdeutschland übergehen, wobei wir zunächst auf die Voraussetzungen der Akkumulation des Kapitals in der BRD, auf die Rolle des Faschismus in Deutschland eingehen werden. Dabei soll der Faschismus nicht nur in seiner Bedeutung für die Entwicklung der Mehrwertrate (durch Lohndruck, erzwungene lange Arbeitszeiten) entwickelt werden, sondern auch als spezifische Lösungsform der stockenden Kapitalbildung in und nach der Weltwirtschaftskrise. Im zweiten Teil dieses Abschnitts werden wir die Phasen der Akkumulation des wesideutschen Kapitals anhand der Untersuchung der Kompo- 
nenten der Kapitalverwertung umreißen. Dabei werden die besonders günstigen Voraussetzungen der Kapitalakkumulation (auf Basis der vorhandenen Potenzen der Wertbildung) in der BRD untersucht (hohe Mehrwertraten, niedriger Kapitalvorschuß) und die Herausbildung der Schranke des ,extensiven ${ }^{6}$ Kapitalwachstums in der Form der Absorption der Lohnarbeiter durch das Kapital. Der Übergang zu veränderten Methoden der Profitproduktion durch die Anwendung neuer Technologie im Produktionsprozeß in der zweiten Phase der Entwicklung des Kapitals wird dann von uns insbesonders auf die darin angelegte veränderte Verwertungssituation und deren entgegenwirkenden Momenten hin untersucht werden, d. h. es wird die Frage zu stellen sein, inwieweit modifizie rende Momente (Verkauf der Arbeitskraft unter Wert, Staat, Weltmarkt und Inflation) für die Verwertungssituation des Kapitals eine besondere Bedeutung gewinnen.

Es ist auch dieser Zusammenhang, der als Bewegungsform der sich entwickelnden Überakkumulationstendenzen in der dritten Phase der Kapitalentwicklung zu untersuchen ist: Wir werden darin versuchen, einige Aspekte des Zusammenhangs von Inflation und stagnativen Tendenzen aufzuzeigen. Darüberhinaus werden die Wirkungen von staatlicher Intervention und der Stellung des westdeutschen Kapitals auf dem Weltmarkt in gesonderten Punkten auf die Verwertungssituation des nationalen Kapitals und deren Schranken resp. Tendenzen umrissen.

Im dritten Abschnitt werden wir dann in einer Gesamtbetrachtung die Entwicklung des Verwertungsgrades des westdeutschen Kapitals anhand der Komponenten der Profitrate darstellen und die dort aufgezeigten Tendenzen anhand der bürgerlichen Statistik zu illustrieren versuchen. Daraus werden dann sowohl Schlüsse zu ziehen sein auf die Entwicklung des zukünftigen Kapitalwachstums und des Waren- und Kapitalexports als auch auf die Entwicklung der Lage der Arbeiterklasse. In diesem Teil soll also auch der bisher im Rahmen der PROKLA besonders vom Redaktionskollektiv Gewerkschaften betonte Zusammenhang von Kapitalakkumulation, Lage der Arbeiterklasse und der Bedingungen der Entwicklung der Arbeiterbewegung weiter materialistisch untermauert werden.

\section{Methodische Probleme der historischen Analyse}

\subsection{Begriffliche und historische Analyse}

Die Darstellung der inneren Organisation einer Gesellschaft ist nicht dasselbe wie die Beschreibung ihrer historischen Entwicklung. Damit die Darstellung der historischen Entwicklungstendenzen nicht begriffslos erfolgt, nicht bloß die Faktizität der Ereignisse konstatiert und allenfalls nach dem Gesetz der Wahrscheinlichkeit Einschätzungen von vergangenen und zukünftigen Entwicklungstendenzen vornimmt, ist es notwendig, die Darstellung der inneren Organisation der bürgerlichen Gesellschaft in ein Verhältnis zu setzen zur Darstellung der historischen Entwicklung - in diesem Fall - Westdeutschlands. Die ganze Schwierigkeit bei der Bestimmung dieses Verhältnisses entsteht deshalb, weil die innere Organisation und die ihr zugrundeliegenden Bewegungsgesetze nicht unmittelbar an der Oberfläche der 
bürgerlichen Gesellschaft erscheinen, sondern nur in vermittelter und verkehrter Form. Diese Verkehrtheit betrifft nicht nur die ,objektiven Verhältnisse ${ }^{66}$, unter denen sich die Gesellschaft reproduziert, sondern auch die Bewußtseins- und Denkformen der Individuen, insbesondere auch deren systematischen Ausdruck, nämlich die bürgerliche Wissenschaft. So ist die wirkliche Geschichte auch der westdeutschen Gesellschaft dem Bewegungsgesetz der kapitalistischen Produktionsweise unterworfen, aber auf der Oberfläche dieser Gesellschaft stellt sich dieses in verkehrten Formen dar und prägt als solches auch das Bewußtsein der Individuen und somit auch ihr Verhalten. ,So leben die Agenten der kapitalistischen Produktion in einer verzauberten Welt... Soweit sich in (der) Bewegung (der Konkurrenz) der innre Zusammenhang durchsetzt, erscheint es als ein mysteriöses Gesetz. Bester Beweis die politische Ökonomie selbst, eine Wissenschaft, die sich damit beschäftigt, den verborgnen Zusammenhang wieder zu entdecken ... "(1)

So wird es notwendig, die Selbstdarstellung dieser, verzauberten Welt ${ }^{\text {cs }} z$ zu dechiffrieren und in den historischen Entwicklungstendenzen das zugrundeliegende Gesetz dieser Entwicklung aufzuspüren. Dies gilt für die Entwicklung der bürgerlichen Gesellschaft schlechthin; dies gilt aber auch für die Untersuchung der westdeutschen Gesellschaft nach dem zweiten Weltkrieg als eine besondere historische Phase dieser Entwicklung. Wenn auch die westdeutsche Gesellschaft den Entwicklungsbedingungen der Kapitalakkumulation schlechthin unterliegt, so doch nicht in dem Sinne, als ob mit der ,,reinen“"Herausarbeitung dieser Bedingungen ,im idealen Durchschnitt" (2) die Tendenzen der Kapitalakkumulation und der gesellschaftlichen Entwicklung hinreichend umschrieben wären. Da es dabei nicht um ein quantitatives Problem der Vollständigkeit z. B. der Datenmaterials geht, sondern es sich um ein qualitatives Problem der Beriucksich tigung des Verhältnisses von allgemeinen Gesetzen, wie sie aus dem Kapitalbegriff im Allgemeinen abgeleitet werden können, und ihren historischen Durchsetzungsformen und -bedingungen in einer historisch bestimmten Gesellschaft handelt, soll darauf hier eingegangen werden.

Die Form der Gesellschaft resultiert aus der Form des Vermittlungszusam. menhangs der gesellschaftlichen Arbeit. In der bürgerlichen Gesellschaft stellt sich dieser Vermittlungszusammenhang nicht planmäßig in von den einzelnen Gesellschaftsgliedern oder ihren gemeinsamen Einrichtungen vorausberechneter und beabsichtigter Weise her, sondern naturwüchsig, resultierend aus den aufeinander bezogenen Aktionen der Produktionsagenten. Die Ergebnisse ihres jeweiligen Handelns entsprechen nicht oder nur zufällig ihren Absichten, so daß ihnen auch Gesellschaftlichkeit als etwas außer ihnen existierendes, ihren Absichten häufig zuwiderlaufendes erscheinen muß. Der Vermittlungszusammenhang gesellschaftlicher Arbeit er. folgt in gegenüber den einzelnen, den Arbeitern und den Kapitalagenten, verselbständigten Formen. Auch die Bewegung der Gesellschaft wird begriffen als verselbständigte Bewegung, die sich ausdrückt in den entsprechenden Tendenzen einzelner

1) Marx-Engels-Werke, Berlin $1956 \mathrm{ff}$. (im folgenden MEW), Bd. 26.3, 3. $503 \mathrm{f}$.

2) MEW, Bd. 25 (Das Kapital, Bd. III), S. 152: , In solcher allgemeinen Untersuchung wird überhaupt immer vorausgesetzt, daß die wirklichen Verhältnisse ihrem Begriff entsprechen, der was dasselbe, werden die wirklichen Verhältnisse nur dargestellt, soweit sie ihren eignen allgemeinen Typus ausd rücken ..." 
Kategorien ais quantitative Vexänderung. Das Wachstum des Sozialprodukts, die Entwicklung der Produk tivität der Arbeit, die Veränderung der Verteilung der Einkommen im Zeitverlauf, die Zunahme der Konzentration der Produktion oder die Veränderungen der Investitionen stellen sich als Bewegungen verselbständigter Formen dar, deren gesellschaftliche Vermittlung nicht mehr in ihnen selbst reflektiert wird. So stellt sich geselischaftliche Veränderung als bloß quantitative Veränderung von Größen dar, die demzufolge auch in Form bestimmter mehr oder weniger rationeli konstruierter ,social indicators" gemessen werden kann. Das diesen Tendenzen zugrundeliegende Gesetz der Entwicklung bleibt verborgen und muß erst. analy tisch herausgearbeitet werden.

Wegen dieses Charakters bürgerlicher Gesellschaftlichkeit und dieser Form, in der sich ihre Entwickling dasstellt, verbietet es sich, die Entwicklungstendenzen der westdeutschen Gesellschaft einfach ohne weitere Voruberlegungen anhand der gängigen Kategorien, in denen sich scheinbar deren Entwicklung reflek tiert, darzustellen. Denn in diesen Kategorien - Sozialprodukt, Produktivität usw.--wird nicht die Wirklichleit kapitalistischer Entwicklung ideell reproduziert, sondem nur deren verkehrte, äußerlichste Formen der Oberfläche reflektiert. Empirisch-historische Analyse zu betreiben, kann demzufolge auch nicht heißen, das diesen Kategorien zukommende Material (in der Regel quantitative, zahlenmäßige Ausdrücke) sczusagen, ,gegen den Strich ${ }^{66}$ zu interpretieren mit dem Interesse, den kritischen Gehalt hervorzukehren. Eine historische Analyse ist erst dann eine Analyse der kapitalistischen Realität, wenn sie auch die Formen, in denen notwendig die, inn nere Organisation " der Gesellschaft und ihre Bewegungsgesetze erscheinen, ableitet und so die oberflächlichsten Formen des erscheinenden Vermittlungszusammenhangs gesellschaftlicher Arbeit bestimmt, um dann zur historischen Analyse der empirischen Bewegung dieser Oberfläche zu schreiten.

Diese methodischen Überlegungen zeigen den hohen Kompliziertheitsgrad einer „Realanalyse“. Sie muß sowohl die Herleitung des „Kapitalbegriffs im Allgemeinen", also auch die Darstellung der notwendig produzierten Erscheinungen bis hin zur Oberfläche der bürgerlichen Gesellschaft, als auch die historische Bewegung dieser Gesellschaft, wie sie sich in den Oberflächenkategorien darstellt und entwickelt, umschließen. Schematisch läßt sich dies wie folgt zusammenfassen. (Natürlich leistet das nachfolgende Schema nicht mehr als eine Abkürzung der Argumentation; es steht nicht an Stelle dieser Argumentation selbst. 
Totalität der bürgeriichen Gesellschaft

\begin{tabular}{|c|c|c|c|}
\hline & $\begin{array}{l}\text { Innere Organisation } \\
\text { (Wesen) }\end{array}$ & $\begin{array}{l}\text { Notwendige äußere } \\
\text { Erscheinungen }\end{array}$ & $\begin{array}{l}\text { Historische Erschei- } \\
\text { nungen }\end{array}$ \\
\hline $\begin{array}{l}\text { Wirkliche } \\
\text { Verhält- } \\
\text { nisse }\end{array}$ & $\begin{array}{l}\text { Gesetze und Tendenzen } \\
\text { der kapitalistischen Pro- } \\
\text { duktionsweise }\end{array}$ & $\begin{array}{l}\text { Kategorien der Ober- } \\
\text { fläche der bürgerli- } \\
\text { chen Gesellschaft }\end{array}$ & $\begin{array}{l}\text { Empirische Bewegung } \\
\text { der Oberfläche der } \\
\text { bürgerlichen Gesellsch. }\end{array}$ \\
\hline \multirow{3}{*}{$\begin{array}{l}\text { Ebenen } \\
\text { der Dar- } \\
\text { stellung } \\
\text { der wirk- } \\
\text { lichen Ver- } \\
\text { hältnisse }\end{array}$} & $\begin{array}{l}\text { Begriffliche Entfaltung } \\
\text { der logischen Struktur } \\
\text { des Kapitalbegriffs im } \\
\text { Allgemeinen }\end{array}$ & $\begin{array}{l}\text { Erscheinungen des } \\
\text { Bewegungsgesetzes } \\
\text { und seiner Durch- } \\
\text { setzungsformen } \\
\text { Darstellung der Er- } \\
\text { scheinungsformen, } \\
\text { der Konkurrenz, der } \\
\text { Oberfläche }\end{array}$ & \multirow{2}{*}{$\begin{array}{l}\text { Darstellung histori- } \\
\text { scher Prozesse in ihrer } \\
\text { Spezifik } \\
\text { Illustrationen von Ge. } \\
\text { setzen und ihren Er- } \\
\text { scheinungsformen }\end{array}$} \\
\hline & \multicolumn{2}{|c|}{$\begin{array}{l}\text { Analyse der Formen der } \\
\text { bürgerlichen Produktionsweise }\end{array}$} & \\
\hline & $\begin{array}{l}\text { (z. B. das Kapital und } \\
\text { seine Widersprüche; Ver- } \\
\text { hältnis von Widerspruch } \\
\text { und Krise) }\end{array}$ & $\begin{array}{l}\text { (z. B. Momente und } \\
\text { Erscheinungsformen } \\
\text { der Krise im Repro- } \\
\text { duktionsprozeß des } \\
\text { des Kapitals) }\end{array}$ & $\begin{array}{l}\text { (z. B. Die Entwicklung } \\
\text { des Krisenzyklus nach } \\
\text { dem 2. Weltkrieg in } \\
\text { Westdeutschland) }\end{array}$ \\
\hline
\end{tabular}

Der hohe Kompliziertheitsgrad der „Realanalyse“ indiziert ihre Schwierigkeit. Und so kommt es, daß häufig die Ebenen der Darstellung getrennt bleiben oder gar „,arbeitsteilig" bearbeitet werden, ohne daß ihr Zusammenhang noch hergestellt würde. Auch wir können hier nicht alle Ebenen der „,Realanalyse " gleicherweise in die Untersuchung der Bedingungen der Kapitalakkumulation in Westdeutschland einbeziehen. Uns kommt es darauf an, den methodischen Stellenwert einer solchen Untersuchung zu verdeutlichen. Aber damit sind keineswegs alle Schwierigkeiten bezeichnet, wenn ein historischer Zeitabschnitt konkret untersucht werden soll. Denn bislang ist lediglich die historische Bewegung der bürgerlichen Gesellschaft, wie sie an der Oberfläche erscheint, benannt und der Stellenwert ihrer abgetrennten Untersuchung methodisch festgelegt. Damit ist jedoch noch nicht herausgearbeitet, wie die Analyse dieser Oberfläche selbst zu erfolgen hat. Hier ergiebt sich noch eine zusätzliche systematische Schwierigkeit. Alle Begriffe, mit denen bei der begrifflichen Explikation der Logik des Kapitals gearbeitet wird, können in der Anschauungswelt bürgerlicher Geschichtsschreibung, in den Datensammlungen der Statistik oder in empirischen Untersuchungen der Sozialforschung nicht unmittelbar aufgefunden werden. Darin spiegelt sich nicht nur das allgemeine Problem, daß die Kategorien des Kapitalverhältnisses nur in mystifizierter Form zu erscheinen vermö- 
gen - der Wert der Arbeitskraft im Lohn oder im ,Einkommen aus unselbständiger Arbeit", der Mehrwert im Profit oder dem „Einkommen aus Unternehmertätigkeit und Vermögen" - und die mystifizierenden Kategorien nicht einfach als Illustrationen oder Indikatoren für ,Wesenskategorien ${ }^{66}$ genommen werden können. Darin kommt auch das spezifische Problem der bürgerlichen Darstellungen zum Ausdruck, daß - weil die Oberflächenkategorien nicht systematisch hergeleitet sind - nur das Mittel der Definition bleibt: Dic jeweiligen Kategorien, mit denen wir auch im folgenden zu operieren haben, sind definiert, $d . h$. je nach dem begründeten Belieben des Forschers oder aufgrund von Konvention festgelegt. Wenn also im folgenden mit Begriffen operiert wird, so immer mit sozusagen doppelt ungenauen Kategorien: sie sind notwendigerweise Verkehrungen der Wesenskategorien und als Verkehrungen noch der definierenden gesellschaftlichen Konvention verdankt. So führen die Kategorien der Oberfläche noch ein "Eigenleben", das nicht in allen seinen Ausdrucksformen den zugrundeliegenden Verhältnissen geschuldet ist. Dies stellt sich insbesondere auch in den wissenschaftlichen Untersuchungen heraus, die in das Chaos der erscheinenden Oberfläche ,Ordnung ${ }^{6} \mathrm{zu}$ bringen versuchen, indem sie klassifizieren und definieren, Beziehungen konstruieren und explizieren und dabei nach unterschiedlichsten Kriterien vorgehen, die es dann demjenigen, der sich auf ihre Untersuchungen zu stützen hat, so schwer machen, den Grad an Verallgemeinerbarkeit zu bestimmen. (So ergeben sich regelmäßig Schwierigkeiten beim internationalen Vergleich von Daten aufgrund national verschiedener Definitionen der jeweiligen Größ3en oder bei der Analyse von Zeitreihen, weil sich inzwischen die Definition der entsprechenden Größen geändert haben mag.)

Angesichts dieser der Oberfläche als Oberfläche der bürgerlichen Gesellschaft geschuldeten zusätzlichen Schwierigkeiten zeigt es sich, daß die methodische Bestimmung des Übergangs von logischer Explikation zu historischer Analyse noch nicht ausreicht, um die Probleme der Realanalyse zu lösen. Es geht hier also um zwei Dimensionen des Problems vor denen jede historische Analyse steht: Einmal hat sie sich der Verkehrungen, in denen die innere Organisation der Gesellschaft erscheint, zu vergewissern. Dabei ist zu berücksichtigen, daß zwischen grundlegenden Kategorien (z.B. dem Wert) und erscheinenden Kategorien (z. B. dem Preis) kein einfaches Verhältnis der Transformation besteht. Denn da Werte nicht erscheinen, und quantitativ meibar nur das ist, was erscheint, sind Werte statistisch prinzipiell nicht meßbar und nur ihre Erscheinungsform als Preis ist faßbar. Die äußerliche Kategorie des Marktpreises liegt somit den statistischen Daten, mit denen wir es in der historischen Analyse zu tun haben, zugrunde. Der Marktpreis und seine Bewegung allein ist meßbar, nicht jedoch die Kategorien, die seine Bewegung letztlich regulieren. Es geht also nicht nur darum, daß die Werte nicht meßbar sind, sondern auch darum, daß nicht einmal der Produktionspreis, in den der Wert unter kapitalistischen Verhältnissen ,transformiert" wird, als solcher in den statistischen Datensammlungen erscheint. Von den Marktpreisen, deren Tendenz und aktuelle Höhe einer Vielzahl von je besonderen Bedingungen geschuldet sind, kann daher nur unter der Annahme auf die zugrundeliegende Wertbewegung rückgeschlossen, daß über eine bestimmte Zeitspanne kalkuliert deren Tendenzen von der Bewegung des Wertes bzw. des Produktionspreises reguliert wird und daher sich in den Markt- 
preisen die Produktionspreise reflektieren. In dieser Annahme sind natürlich eine ganze Reihe von Problemen eingeschlossen. Denn nun wird bei der statistischen Illustration von historischen Tendenzen genau umgekehrt vorgegangen als in der logischen Analyse: Während in der begrifflichen Explikation die äußerlichsten Kategorien ,abgeleitet" werden und als erscheinende Oberfläche aus der Struktur des Kapitals resultieren, werden in der historischen Analyse die äußerlichsten Kategorien, in diesem Fall die Marktpreise, ,benutzt ${ }^{66}$, um Entwicklungstendenzen der Reproduktionsstruktur des Kapitals zu dokumentieren. Dies sist, um es noch einmal zu wiederholen, nur unter der allerdings begründeten Annahme möglich, daß sich in der Bewegung der äußerlichsten Kategorien die Wertbewegung doch reflektiert und als solche also in dem Material, das in Marktpreisen ausgedrückt ist, interpretiert werden kann. Denn auch bei der Bewegung der Marktpreise muß sich letztlich die Tatsache Geltung verschaffen, daß der Gesellschaft nur ein sozial begrenztes Arbeitsquantum zur Verfügung steht, welches zugleich in richtigen Proportionen auf die verschiedenen Produktionszweige sich verteilen muß. Dieses von Marx formulierte Naturgesetz der gesellschaftlichen Produktion kann auch durch die wie auch irnmer bewirkten Marktpreisbewegungen nicht aufgehoben werden, sondern macht sich als Begrenzung ihrer scheinbar freien Schwankungen geltend. Welche Probleme sich gerade dadurch - in bezug auf unser Problem der empirisch-statistischen Erfassung der Wertbewegung - ergeben, kann eine kurze Überlegung hinsichtlich der Messung der Wachstumsrate des Sozialprodukts klarmachen. Entsprechend der Entwicklungstendenz des Kapitals, die Produktivkraft der Arbeit zum Zweck der Produktion relativen Mehrwerts permanent zu erhöhen, sinkt die gesellschaftlich notwendige Arbeitsmenge, die zur Produktion einer bestimmten Produktmenge aufgewendet werden muß, bzw. steigt die Produktenmenge entsprechend der Produktivkraftsteigerung bei gleichm Arbeitsvolumen. Die in einem Zeitraum, z. B. in einem Jahr, aufgewendete Arbeitsmenge ist nach dieser Annahme nicht gestiegen, wohl aber die produzierte Menge von Gebrauchswerten. Wie selbstverständlich wird die gestiegene Menge Gebiauchswerte mit dem jeweiligen Preis der Waren multipliziert und ergibt dann als Resultat das Bruttosozialprodukt. In der bürgerlichen Ökonomie wird dieses Bruttosozialprodukt nun mit zwei möglichen Preisen alternativ bewertet. Entweder zu laufenden Marktpreisen, oder aber zu den Marktpreisen einer vorhergehenden Periode. Bei der Bewertung zu laufenden Marktpreisen ist in der Größe des jeweiligen Jahressozialprodukts die "Inflationsrate" enthalten; bei der Bewertung zu den Marktpreisen der Vorperiode ist diese "Inflationsrate" statistisch ausgeschaltet und das so gemessene Wachstum stellt nach Ansicht der Ökonomen das ,reale "Sozialprodukt dar und seine Steigerung gegenüber der Vorperiode dementsprechend die ,reale" Wachstumsrate.

Ob aber nominales oder reales Wachstum gemessen wird, immer ist die me tho. dische Grundlage die gleiche: die Masse produzierter Gebrauchswerte wird mit dem entsprechenden Marktpreis, dem gegenwärtigen oder demjenigen einer vergangenen Periode multipliziert. So geht dann verloren, daß ja gerade aufgrund der Produktivkraftsteigerung der Arbeitsaufwand pro Wareneinheit gesunken ist und daher zwar der reale Reichtum einer Nation in Gestalt des gestiegenen Gebrauchswertequantums gestiegen ist, aber der Wert der einzelnen Ware (im Durchschnitt) gesunken 
sein muß. Daraus ergibt sich, daß die Marktpreise in ihrer absoluten Bewegung dann nicht mehr die Wertbewegung reflektieren, wenn durch inflationistische Prozesse die Wertsenkung der einzelnen Ware nicht mehr aus ihren Ausdruck als Preissenkung findet. Werner Hofmann hat diese Tatsache als relative Inflation bezeichnet. Selbst konstante Preise sind so noch Ausdruck inflationistischer Tendenzen. Die Marktpreise sind Einflüssen unterworfen, die nicht allein aus der Wertbewegung der einzelnen Ware stammen, sondem den Bedingungen der Kapitalakkumulation (z. B. darin eingeschlossenen Angebots- und Nachfrageverhältrissen, Kreditinflationen etc.) geschuldet sind. (Darauf soll noch ausführlich im zweiten Abschnitt eingegangen werden.) Dies betrifft sowohl die Berechnung des Sozialprodukts und seiner Wachstumsrate in laufenden als auch diejenige in konstanten Preisen.

Die statistische Ausschaltung der ,,relativen Inflation ${ }^{66}$ ist in der bürgerlichen Statistik unmöglich aus zwei Gründen: Erstens ist, darauf wurde schon kurz hingewiesen, das Messen des Sozialprodukts in monetären Größsen selbst ein Ausdruck der spezifischen Auffassung von Gesellschaftlichkeit als eines sich in bestimmten Kategorien ausdrückenden äußerlichen Zusammenhangs und nicht als kon tinuiertich reproduziertes Resultat gesellschaftlicher Arbeit. Insofern wird auch der gesellschaftliche Reichtum in seiner ve rselbständigten Gestalt, als in Marktpreisen quanti. fizierter "Kuchen" vorgestellt; daß dieser „Kuchen" das Resultat gesellschaftlicher Arbeit ist und seine Vergrößerung nur die Gebrauchswertseite, und nicht die Wertseite betrifft, bleibt so verborgen. Zweitens würde die Ausschaltung der relativen Inflation notwendig zu der Erkenntnis führen, daß das gesellschaftliche Nettopro. dukt (Wertprodukt) allein Produkt gesellschaftlich verausgabter Arbeit ist. Dies aber konfligiert mit den Produktionsfaktoren-Theorie, derzufolge das gesellschaftliche Nettoprodukt Resultat des vom Untemehmer besorgten Kombinationsprozesses von Arbeit, Kapital und Boden darstellt. Gerade die Bewertung des Produktenquantums mit dem Marktpreis macht es möglich, daß die in die Pxoduktion wieder eingehenden Produktionsagentien wertbildende Kraft zu gewinnen scheinen. So zeigt es sich, wie problematisch die unbefragte Verwendung der Kategorien des Soziaiprodukts und seiner Steigerungsraten ist.

A ber nicht genug damit. Die quantitativen Ausdricke von bestimmten Kategorien, die entsprechenden Daten also, werden in der Regel bei Einzelkapitalisten erhoben und aggregiert. Die einzelnen Unternehmen aber haben unter Umständen gar kein Interesse daran, strategisch wichtige Iinformationen, „richtig“ anzugeben (3), ganz abgesehen davon, daß ihnen selbst gnindlegende kategoriale Bestimmungen dunkel sind, z. B. die Unterscheidung von produktiver und unproduktiver Arbeit bei der Angabe von Produktivitätsziffem. Schließlich darf auch nicht vergessen werden, daß die Daten in der Regel von Einrichtungen des bürgerlichen $S$ taates (dem Statistischen Bundesamt) erhoben werden, dessen Interesse an ausführlicher Datenerhebung und Dokumentation in bestimmter begrifflicher Ordnung ihm nicht von $N$ atur aus mitgegeben ist, sondern ihm immer wieder, aufgeherrscht ${ }^{\text {t6 }}$ werden

3) In einem Rundschreiben des Verbands der südbadischen Industrie während des Metallarbeiterstreiks 1971 heibt es: ,Die ,Zahlen' sind Ausgangsbasis für die unternehmerische Öffentlichkeitsarbeit. Sie sind unbestechlich, handlich aufbereitet und zweckentsprechend ausgesucht." Mit Zahlen läßt sich also trefflich streiten ... 
muB, wie die Erstellung von spezifischen Statistiken, z. B. zur Vermögenskonzentration, zur Einkommensverteilung, zur Qualifikationsstruktur, zeigt (4).

Über diese Schwierigkeiten muß man sich bei der historischen Analyse eines bestimmten Zeitabschnittes der Kapitalakkumulation im klaren sein. Es sind Schwierigkeiten, die nicht nur aus der Vermittlung von innerer Organisation der Gesellschaft und ihrer Oberfläche stammen, sondern auch aus der Nicht-Struktur dieser Oberfläche selbst. So ergibt es sich als eine Aufgabe der historischen Analyse, diese unstrukturierte Oberfläche noch als solche zu strukturieren. Dies scheint Marx im Auge gehabt zu haben, als er in einem Brief an Engels im Jahre 1873 schrieb:

„... die Sache ist die: Du kennst die Tabellen, worin Preise, Discountrate etc., etc. in ihrer Bewegung während des Jahres erc. in auf- und absteigenden Zickzacks dargestellt sind. Ich habe verschiednemale versucht - zur Analyse der Krisen -, diese ups and downs als unregelmäßige Kurven zu berechnen und geglaubt (ich glaube noch, daß es mit hinreichend gesichertem Material möglich ist), daraus die Hauptgesetze der Krisen mathematisch zu bestimmen. Moore, wie gesagt, häit die Sache einstweilen für untubar, und ich habe beschlossen, for the time being es aufzugeben" (5).

Diese Bemeikung könnte durchaus von einem bürgerlichen Konjunkturforscher stammen, der anhand von Symptomen der Konjunkturbewegung versucht, längerfristige Zeitreihen aufzustellen und deren Gesetzmäßigkeiten induktiv zu erforschen. ,Die Methode besteht einfach darin, Tatsachen - wenn möglich Zahlen - zu beobachten, die solche Phänomene betreffen, von denen wir aus Erfahrung wissen, daß sie in sich selbst oder aus Erfahrung wichtig sind ... (6). Viele solcher Symptome zusammengenommen ergeben ein ,Konjunk turbarome ter ${ }^{66}(7)$, dessen Ausschläge die wirklichen ,ups and downs" der Kapitalakkumulation anzeigen soll. Hier ist keine Vermittlung mehr zu den Ursachen dieser ,ups and downs" angelegt, es werden lediglich die Bewegungen einiger symptomatischer Kategorien indiziert. Das

4) Es wäre mehr als interessant zu untersuchen, unter welchen konkreten Umständen jeweils das statistische Kategoriensystem verändert wurde, warum überhaupt das Bedürfnis nach quantitativer Ermittlung der Struktut und Bewegung der bürgerlichen Gesellschaft und ihrer Wirtschaft entstand, wie bestimmte Methoden sich herausbildeten usw. Ein Beispiel dafür sei aus dem Jahresgutachten des \$achverständigenrates (SVR) zur Begutachtung der gesamtwirtschaftlichen Entwicklung von 1973 (JG 73) zitiert. Bei der Behandiung der Mietpreissteigerungen kommt der SVR zu dem Ergebnis, daß Mietpreissteigerungen nur noch möglich waren aufgrund der inflationsbedingten, ,Flucht in die Sachwerte". Sollten die inflationären Prozesse sich verlangsamen oder gar aufhören zu wirken, so lautet seine These, dann würde sich herausstellen, daß Kapital volkswirtschaftlich verschwendet worden sei, ,weil es dorthin gelenkt wurde, wo nicht die größten Erträge, sondern nur die größten Inflationsgewinne erwartet wurden." (JG 73, Ziff, 102) Und der SVR fähr dann fort: ,Zu diesem problemkreis liefert die amtliche Statistik kaum Informationen ... " (Ziff. 103) Mit anderen Worten: Erst mußs sich ein Problem als problematisch dem Bewußtsein aufdrängen, bevor es statistisch untersucht werden kann. Ähnliches ließe sich etwa zur Vermögens- und Konzentrationsstatistik aussagen.

5) MEW, Bd. 33, S. 82

6) Joseph A. Schumpeter, Konjunk turzyklen, Bd, 1, Göttingen 1961, S. 21

7) Das Harvard-Institut war eines der ersten, die ein sogenanntes Konjunkturbarometer erstellten. Vgl. dazu Rudolf Gater, Die Konjunktur-Prognose des Harvard-Institutes, Zürich 1931 und Geoffrey H. Moore, Statistical Indicators of Cyclical Revivals and Reces sions, New York 1950 
Scheitern der bürgerlichen Ökonomie bei diesem Unterfangen aber deutet schon an, daß es so nicht geht, und daß schließlich auch die Marusche Bemerkung so nicht gemeint sein kann. Aber in ihr wird der Versuch ausgedrückt, nicht nur die Oberflächenkategorien und ihre Bewegung abzuleiten, sondern in der Oberflächenbewegung selbst noch Gesetzmäßigkeiten auszumachen, die in den Kategorien der Oberfläche auszudrücken sind und nicht in Wertbegriffen. Letztlich handelt es sich hier um einen Versuch, Indikatoren auf der Oberfläche der bürgerlichen Gesellschaft und ihrer Entwicklung in der systematischen Beziehung und Entwicklung für die zugrundeliegende Wertbewegung auszumachen, die ja in handfesten quantitativ meßbaren Größen nicht vorliegt. So ergibt sich die schwierige Aufgabe, die Oberfläche der bürgerlichen Gesellschaft nicht als etwas Selbständiges und Absolutes zu behandeln. Denn

,die Erscheinungen werden zur phänomenalen Welt kraft ihrex Beziehung zum Wesen ... Die Erscheinung einer bestimmten Sache erfassen, bedeutet erforschen und beschreiben, wie die Sache selbst sich in dieser Erscheinung offenbart, aber auch wie sie sich gleichzeitig verbirgt. Im Erfassen einer Erscheinung verschafft man sich Zutritt zum Wesen ... Die Wirklichkeit ist die Einheit von Erscheinung und Wesen" (8).

Die innere Struktur der Erscheinungen selbst aber stell $t$ sich in Beziehungen der einzelnen Erscheinungen untereinander auch dar.

„Die Oberflächenschicht dieses Gesamtprozesses ist ein Netz von Kausalbeziehungen zwischen diesen einzelnen Momenten als verselbständigten Faktoren ... Die einheitliche, ganzheitliche, innerlich widersprüchliche Bewegung enthält in sich eine Vielzahl einzelner Momente, die sich gegeneinander verselbständigen und zueinander in Kausalverhältnisse treten . . " (9).

Damit ist bereits angedeutet, worum es sich handelt: Es geht darum, in der Bewegung der Oberfläche und ihrer Kategorien die "Grundbewegung" (10), d. h. die Wertbewegung und deren, ,innere Logizität ${ }^{66}$, sowie den Reflex dieser Grundbewegung in der Vielzahl von Kausalbeziehungen der Kategorien an der Oberfläche der bürgerlichen Produktionsweise aufzudecken. So verstanden ist der Versuch, ,die ups and downs als unregelmäßige Kurven zu berechnen", auch keineswegs absurd oder der bürgerlichen Symptomatologie gleichzusetzen, solange die erscheinenden Kausalbeziehungen der Oberflächenkategorien als verselbständigte Formen untereinander als Reflex der Wertbewegung und ihrer Gesetzmäßigkeit begriffen werden.

Diese Überlegung kann an einem Beispiel verdeutlicht werden. Wir gehen davon aus, daß in einer Phase guter Konjunktur bei Vollbeschäftigung die Arbeiterklasse durch Lohnkämpfe nicht nur ihre Reallöhne hat anheben, sondern auch die Verteilungsverhältnisse $z$ wischen den Klassen $z u$ ihren Gunsten hat verändern können. Dies mag negative Konsequenzen für die Kapitalverwertung haben: die Profitrate, die während des Aufschwungs angestiegen ist, mag nun fallen. Einzelne Kapitale drosseln die Produktion, oder gehen gar bankrott; eine ökonomische Krise deutet sich an und kommt tatsächlich mit Zeitverzögerung zum Ausbruch. Diese

8) Karel Kosik, Die Dialektik des Konkreten, Frankfurt a. M. 1967, S. 10

9) J. Cibulka, zit. bei Jindrich Zeleny, Die Wissenschaftslogik und das ,Kapital', Frankfurt und Wien 1968, S. 110

10)

Ebd. 
Grundbewegung des Widerspruchs von Lohnarbeit und Kapital findet Ausdrucksformen an der Oberfläche der bürgerlichen Gesellschaft und natürlich im statistischen Material: Die Reallöhne steigen, die "Realrendite der gewerblichen Wirtschaft $t^{\text {ts }}$ sinkt, die Preise für Konsumwaren der Arbeiterklasse steigen aufgrund der wachsenden Nachfrage noch, während der Absatz von Produktionsmitteln bereits stockt und mit Preissenkungen einhergeht. Die Zinsrate stejgt, da die Untemehmen Zirku lationskredite brauchen. Die Neuinvestitionen weisen abnehmende Zuwachsraten auf, bis sie auf Null zurückgehen. In den Oberflächenerscheinungen drückt sich folglich die Grundbewegung ,irgendwie ${ }^{\text {"s }}$ aus; und die einzelnen Momente dieser Oberfläche scheinen in Kausalbeziehungen zueinander zu stehen. Dieser Schein, der Anlaß gibt zu der Vielzahl von Konjunkturmodellen, die in der Konjunkturtheorie bislang konstruiert worden sind, kann aber nur entstehen, weil die einzelnen Momente der Oberfläche in ihrer Existenz und Bewegung durch die, innere Logizität der Wertbewegung ${ }^{66}$ bedingt sind. Die Kausal oder Funktionalberiehungen der Momente der Oberfläche untereinander sind nur ein Reflex dieser Redingtheit. Aber eingedenk dieser Tatsache kann marn daran gehen, in den Beziehungen der Momente aufeinander die Tendenzen der Grundbewegung, die sich quantitativ nicht messen läs $t$, herauszubekommen. Dies jedenfalls scheint Mark mit seinen Versuchen zur Berechnung der Konjunkturschwankungen beabsichtigt zu haben.

$\checkmark$ on diesem Punkt versuchen wir in der folgenden historischen Analyse der westdeutschen Kapitalakkumulation auszagehen. Wenn die Kapitadkkmulation sich als Bewegung sowohl der, grundlegenden ", also nicht als solche an der Obernäche erscheinenden Kategorien, als auch der erscheinenden Oberifache dasstellt, dann hat diese Unterscheidung Konsequenzen für die Verwendung von empirischem Material. Denn đie grundlegenden Bewegungen können empirische Materialien, z, B. statistische Daten nur illustrieren, die Bewegungen der Oberflächenkategorien aber als solche darstellen, was einen Unterschied macht. Um ein Beispiel zu geben: Anhand sinnvoll ausgewühlter Daten kann somit die als solche nicht an der Oberfläche erscheinende Bewegung der Mehrwertrate illustriert werden, während die Preisbewegung der in der Reproduktion der Arbeitskraft eingehenden Konsumwaren als solche dargesiellt werden können. Wir betonen diese Unterscheidung, um von Anfang an zu verdeutlichen, daß wir statistisches Material in zwei verschie denen Weisen benutzen, deren Verschiedenheit der Unterscheidung von Wesen und ersckeinendem Wesen geschuldet ist. Mlustrationen können niemals die illustrierten Kategorien als solche darstellen. Aber wir benutzen sie, um an ihren Veränderungen im Zeitverlauf auf die historischen Veränderungen, und vor aliem auf die Richtung der Veränderung, der illustrierten Kategorien zu verweisen (11).

11) Wir folgen damit einem Vorgehen von Marx, der beispielsweise im 5. Unterabschnitt ces 23. Kapitels vom ersten Band des, Kapital" "Das allgemeine Gesetz der kapitalistischen Akkumulation" sehr ausführliche „Illustration des allgemeinen Gesetzes der kapitalistischen Akkumulation" gibt. 


\subsection{Empirische Indikatoren für Wertveränderung und Kapitalverwertung}

Der Versuch, die wichtigsten Momente, die die einzelnen Phasen der Entwicklung des Kapitals in einem einzelnen Land bestimmen, in ihren inneren Zusammenhang zu stellen, hat - wie jede Analyse des kapitalistischen Akkumulationsprozesses von der Untersuchung zweier der historischen Entwicklung des Kapitals zugrundeliegenden Faktoren auszugehen:

Es sind die Bedingungen, unter denen sich der Wertbildungsprozeß vollzieht, und die Faktoren der Wertbildung selbst in ihrer Entwicklung, und

es sind die Momente, die den Prozeß der Reproduktion und Erweiterung des Kapitals bestimmen, also im wesentlichen die Komponenten der Profitrate (und damit der ,Motor" der Kapitalakkumulation) zu untersuchen;

- $\quad$ es ist im weiteren zu fragen, wie Wertbildungsprozeß und Kapitalverwertungsprozeß miteinander vermit telt sind.

Um den kategorialen Rahmen, innerhalb dessen sich unsere historische Untersuchung vollziehen wird, zu präzisieren und um weiterhin die Möglichkeit der Verwendung von Indikatoren, die aus der bürgerlichen Statistik entnommen worden sind, aufzuzeigen und deren beschränkten Aussagewert zugleich darstellen zu können, werden wir im folgenden dieses wechselseitige Verhälınis von Wertbildungs- und Verwertungsprozeß $B$ anhand ihrer wichtigsten Bedingungen und Komponenten verdeutlichen und dabei die Kategorien der bürgerlichen Statistik auf die von Marx entwickelten inneren Zusammenhänge beziehen (12).

Der Wert einer Ware bemißt sich durch die gesamte in dieser enthaltenen Arbeit, die $2 u$ herrschenden Durchschnittsbedingungen (gesellschaftliche Durchschnittsbedin. gungen der Produktion, Durchschnittsintensität, Durchschnittsgeschicklichkeit - Qualifikation -) verausgabt sein muß. Andererseits muß diese aber auch auf ein gesellschaftiches Bedurfnis treffen, d. h. die produzierte Ware muß verkaufbar, ihr Wert muß realisierbar sein, wenn die darin enthaltene Arbeit gesellschaftich notwendige gewesen sein soll. Diese hier anhand der beiden Bestimmungen des Begriffs "gesellschaftlich notwendig" kurz angerissenen Bedingungen des Wertbildungsprozesses werden im folgenden vorausgesetzt und wir werden später bei der Analyse des Zusammenhangs von Weltmarkt und Wertbildung in nationalen Rahmen darauf explizit zurückkommen. Geht man nun mit Marx davon aus, daß allein die Verausgabung von Arbeit Werte schafft, so ist der Wertbildungsprozeß offensichtlich von

12) Wenn wir im folgenden aus der Bewegung der Marktpreise auf die Wertbewegung schlieben, so sind wir uns des methodischen Mangels, der darin liegt, daß das Verhälnis von Marktpreisen zu Produktionspreisen (bzw. Marktproduktionspreisen) hierin nicht reflektiert wird, bewulst. Die hier noch zu leistende methodische Diskussion soll an anderer Stelle geführt werden. Insofern wir uns aber in unserer späteren empirischen Analyse auf die gesamtgesellschaftliche Bewegung der Summe der Marktpreise beziehen werden, glauben wir, daß dieser methodische Mangel für unsere weiteren Untersuchungen hier nicht bedeutsam ist. 
der Menge der verausgabten Arbeit abhängig und damit von den einzelnen Komponenten der angewandten Arbeitsmasse:

der Zahl der im Produktionsprozeß angewandten (und zwar - kapitalistisch gesehen - produktiv angewandten) Arbeit, wenngleich auch die nicht unter dem Kommando eines Kapitalisten arbeitenden kleinen Warenproduzenten natürlich Werte produzieren,

- der Zahl der von diesen Arbeitern geleisteten Arbeitsstunden,

- der Intensität der Arbeit, soweit diese noch nicht verallgemeinert ist und insofern noch extensiv wirkt, damit also Moment des Wertbildungsprozesses ist. (Diese Komponente des Wertbildungsprozesses wirđ im folgenden außser acht gelassen.)

Das von einer Nation (z. B. jährlich) produzierte Wertprodukt wird somit durch die Anzahl der im Produktionsprozeß angewandten Arbeiter multipliziert mit der pro Arbeiter geleisteten jährlichen Arbeitszeit gebildet. Die Wachstumsrate dieser Wertmasse ist also abhängig von der Zunahme der angewandten Arbeiter und der pro Arbeiter geleiste ten Arbeitszeit.

Betrachtet man das Wertprodukt als allein abhängig von der insgesarnt verausgabten Arbeit als einziges wertbildendes Moment (quantitativ bestimmt durch die Arbeitszeit), so könnte diese Beziehung in der folgenden Form ausgedrückt werden: ( $N$ gleich Anzahl der Arbeiter, L gleich geleiste te Arbeitszeit pro Arbeiter, Y gleich dem Nettoproduktenwert)

$$
\frac{\Delta(\mathrm{LN})}{(\mathrm{LN})} \approx \frac{\Delta \mathrm{Y}}{\mathrm{Y}}
$$

Warum diese Beziehung nur mit Einschränkungen gilt - da sich in der bürgerlichen Reclnung in der Veränderung des Nettoprodukts auch die Veränderung der Produk tivität ausdrückt - werden wir im folgenden analysieren (12a).

Wird nämlich in der amtlichen Statistik das Arbeitsvolumen konstant gesetzt,

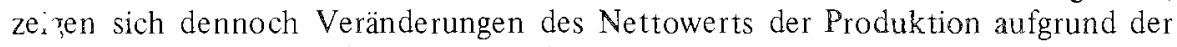
Entwicklung der Produktivität, also durch die Steigerung der physischen Outputs pro eingesetzter Arbeitskraft bzw. pro Arbeitsstunde. Die amtliche Statistik mißt also e twa im Bruttoinlandsprodukt (BIP) nur die Veränderung der Gebrauchswertmengen (13). Dieses Problem wollen wir im folgenden näher analysieren: Das nominelle BIP wird in folgender Weise berechnet:

Umsatz der Unternehmen

\pm Lagerbestandsveränderungen

- Vorleistungen

Bruttoinlandsprodukt

12a) Ausgangspunkt soll der in der bürgerlichen Statistik erfaste Produktionswert aller Wirtschaftsbereiche, das Bruttoinlandsprodukt, sein. Es soll der Frage nachgegangen werden, welcher Zusammenhang zwischen dem Wachstum der Wertmassen dem Wachstum des Bruttoinlandsprodukts besteht und ob ein Wachstum der Wertmassen in dem Wachstum des BIP ausgedrüickt wird. 
Daraus ergibt sich, daß das BIP nicht mit dem Wertprodukt und den vergegenständlichten Wertmassen übereinstimmen kann, da

- die (im Zyklus stark flukturierenden) Abschreibungen (d. h. der Kapitalersatz) noch im BIP enthatten sind,

- die Berechnung des nominellen BIP auf der Basis von Marktpreisen geschieht, d. h. daß die zufällig durch Angebot und Nachfrage bestimmten Preisausdrücke des Werts dieser Berechnung zugrunde liegen, nicht aber Wertquanta und noch nicht einmal Produktionspreise,

- das'BIP - indem es zugleich auf die Gebrauchswertmenge bezogen ist - allenfalls das Niveau der Produktivität und Intensität der Arbeit widerspiegelt und daher zunächst in keinem unmittelbaren Zusammenhang zur Veränderung der Wertmassen steht.

Alle Aggregate - wie BIP, Volkseinkommen, Lohn, Profit usw. - werden in der bürgerlichen Statistik zunächst in nominellen Größen erfaßt, d. h. zu laufenden Preisen. Die Veränderung des nominellen BIP etwa ist deshalb nicht nur bedingt durch die Steigerung der Produktivität und Intensität der Arbeit, sondem auch durch die Veränderung der absoluten Preise oder Waren.

In der Amtlichen Statistik wird zunächst nur der Einfluß der Änderungsrate der absoluten Preisausdrücke der einzelnen Waren eliminiert, indem das BIP mit Hilfe eines errechneten Preisindex' deflationiert wird. (Auf die Eliminierung des Einflusses der relativen Preise und Mengen wird meist verzichtet.)(13)

In dieser Form drückt also die Entwicklung des BIP wesentlich die Entwicklung der Arbeitsproduktivität, also die Entwicklung der Gebrauchswertproduktion aus. In Bezug auf die Wertbildung lassen sich nun drei Konsequenzen ziehen:

(1) Die Wachstumsrate des BIP ist kein Indikator für die Wachstumsraten der Wertmassen - dem vergegenständlichten gesellschaftlich notwendigen Arbeitsquanta. Ein steigendes BIP kann fallende, gleichbleibende oder steigende Wertmassen ausdrücken. Das BIP spiegelt also nur die Gebrauchswertmengen für den Kapitalersatz sowie für das konsumierbare und akkumulierbare physische Produkt wider.

(2) Die Steigerung des, ,realen" BIP bei gleichbleibenden Preisen drück t bereits einen Inflationsprozeß aus, die ,relative Inflation" (W. Hofmann). Das Wachstum der in das BIP eingehenden Gebrauchswertmengen (als Folge der gesteigerten Produk tivität und Intensität der Arbeit) hat zur Konsequenz, daß die pro Ware und im gesamten aggregierten Warenprodukt enthaltene gesellschaftlich notwendige Arbeit einen höheren Geldausdruck annimmt. Ein steigendes reales BIP hat also einen ,inflationierten“ Geldausdruck der gesellschaftlich notwendigen Arbeit zu Folge. Die gleichbleibende (oder steigende) gesellschaftlich notwendige Arbeit (der Neuwert) ,inflationiert" gewissermaßen bei steigendem BIP mit konstanten Preisen! (14)

(3) Da aber alle Aggregate wie BIP, Kapitalstock, Lohn und Lohnquote in unseren unten zu entwickelnden Formeln auf eine Preisbasis (z. B. von 1962)

13) Vgl, zur Frage der Produktivitätsmessung auch: Klaus Grenzdörffer, Probleme der Produktivitätsmessung in der empirischen Wirtschaftsforschung, in: Das Argument 73/1972

14) Vgl. Karl Marx, Das Kapital, Bd. 3, Berlin (DDR) 1957 (Volksausgabe), S. 957 
deflationiert sind (bzw. ihre darin enthaltenen Arbeitsquanta aufgrund der relativen Inflation inflationiert sind), können trotzdem entsprechende Quo. tienten und Verhältniszahlen - vorbehaltlich des Problems der relativen Werte (Beise) und Mengen - entsprechende Wertverhälnisse wiederspiegeh. Mit diesen Einschränkungen - die unten noch teilweise fortgefuhrt werden kann daher zunächst mit den empirisch-statistischen Größen gearbeitet werden.

\section{b) Der Prozeßs der Kapitalverwertung und des Wachstums des Kapitals}

Wenn wir irn weiteren die einzelnen Komponenten des Verwertungsgrades und die Faktoren des Wachstums des Kapitals untersuchen und den Zusammenhang zwischen ihnen in eine mathematisierte Form bringen, so wird dies im wesenthichen deshalb geschehen, um die widerspriichlichen Tendenzen im Akkumulationsprozes des Kapitals in den verfugbaren Materialien der bügerlichen Statistik aufspüren zu kömen (15).

Während bei der Wertbildung nur die verausagate produktive Arbeit für die Erstellung des Nettoprodukts bzw. Wertprodukts zählt, hängt unter den Bedingungen durchgesetzter kapitalistischer Produktionsweise die kontinuierliche Produktion des Nettoprodukts von der Verwertung des vorgeschossenen Kapitals ab. Die Bedingungen der Verwertung und des Wachstums des Kapitals in einem Land sind -.. gesamtgesellschaftich - durch die Größe des Profits im Verhältnis zum vorgeschosseren Kapital gesetzt; dieser Profit ist wiederum durch das Teilungsverhältnis der in den Waren enthaltenen Arbeit (in bezahlte und unbezahlte) in seiner Größe bedingt. Die angewandte Masse Arbeit, das Verhälinis von unbezahlier Arbeit zur bezahlten Arbeit (Mehrwertrate) und der Wert des vom Kapitalisten vorzuschie'3enden Kapi$t a t^{\prime}$ sind also die wichtigsten Faktoren der Veränderung der Größe der Profitrate (und im weiteren - wie unten noch ausgefuhrt wird - der Rate der Kapitalbildung).

Die Profitrate wollen wir im folgenden so schreiben, daß sowohl der der Ausbeutungsgrad der Arbeit als auch die organische Zusammensetzung des Kapitals sichtbar werden:

$$
p^{\prime}=\frac{m}{v} \cdot \frac{v}{c+v}
$$

$$
\mathrm{p}^{\prime}=\frac{\frac{\mathrm{m}}{\mathrm{v}}}{1+\frac{\mathrm{C}}{\mathrm{V}}}
$$

15) Dabei geht es uns also in erster Linie nicht darum, z. B. zu „,beweisen" daß die Profitiate falt, sondern carum, anhand der Entwicklung des Verwertungsgrades des Kapitals und der darin sichtbaren inneren Tendenzen (etwa zur Qberakkumulation) die Rolle der dem tendenziellen Fall entgegenwirkenden Momente darzustellen und insgesamt die Bewegungsform der Konflikte im Akkumulationsprozefs mit der Lage der Arbeiterklasse und mit den Beơingungen der Klassenauseinandersetzungen aufzuzeigen. Wir haben diesen Zusammenhang bereits an anderer Stelle ausführlich dargestellt (in: Semmler/Hoffmann, Kapitalakkumulation, Staatseingriffe und Lohnbewegung, in: PROKLA 2/1972) und werden urs im folgenden auf die dort entwickelten Zusammenhänge stützen bzw. diese voraussetzen. Andererseits können wir hier, wo es uns darum geht, die entscheidenden Momente dex Wertbildung und Verwertung herauszuarbeiten, nicht auf jeden Aspekt der Kapitalakkumulation eingehen. 
Anhand der Formel (1a) lassen sich alle Voraussetzungen und Bedingungen der Erhaltung des Kapitalverhältnisses, der Reproduktion und Akkumulation von Kapital interpretieren. Dazu gehören auch die quantitativen Großen wie die Rate des Mehrwerts, die Masse des Mehrwerts (durch die uberhaupt angewandten produktiven Arbeiter produziert), die Größe der Kapitalauslagen für die Produktion des Mehrwerts sowie die Bedeutung des konstanten (fixen und zirkulierenden) Kapitals und seines Werts und seiner Verbilligung (sei es durch produktivere Produktionsmethoden, kapitalsparende Innovationen, sei es durch die Weltmarktzusammenhänge). Darüberhinaus ist auch aus diesem Zusammenhang die Bedeutung der perio. dischein Entwertung des Kapitals, die sowohl den Kapitalstock wie auch Lagerbestände und Warenkapital zu entwickeln (16).

In der Schreibweise (1b) der Profitrate erscheint der Verwertungsgrad des Kapitals als abhängige Variable von zwei wesentlichen Quotienten, der Mehrwertrate und der organischen Zusammensetzung des Kapitals.

16) was besonders bei Betrachtung der crsten Phase der Entwicklung des BRD-Kapitals in den $50 \mathrm{er}$ Jahren von Bedeutung sein wird!

\section{Materialien und Untersuchungen zu Kapital- bewegung, Staatsapparat und Klassenanalyse in den Industriezentren der BRD und der EG}

Die Texte, die in dieser Reihe als erste erscheinen, sind Untersuchungen zur politischen Okonomie des Ruhrgebiets; die Titel 2 bis 4 sind im Rahmen der Projektgruppe Ruhrgebietsanalyse, Bochum, entstanden:

+ Teilkrise ohme Ende

+ Opel stroikt!

+ Ruhrstaht und Imperialismus

+ Steinkohlankrise im Ruhrgebiet und der Konkurrenzkampt aut dem Welrenergiemarkt

Das Ruhrgebiet ist auch heute noch eines der wichtigsten Zentren der BRD und EG.

Daher lassen sich an ihm exemplarisch die Entwicklung des Imperialismus, der großen Konzerne und der Banken in Verbindung mit dem Staat sowie die Kämpfe der Arbeiterklasse gegen das Kapital und seinen Staat und gegen ihre "eigenen Vertreter" im Gewerkschaftsapparat aufzeigen.

In dieser Reihe sind ähnliche Analysen über andere Industriezentren der BRD und $E G$ vorgesehen.

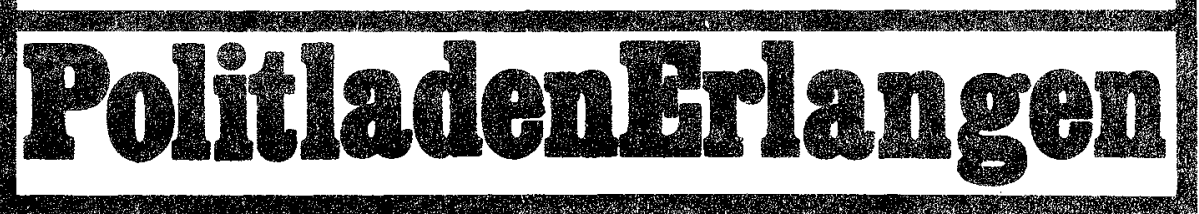


In beiden Schreibweisen stoßen wir aber auf die Schwierigkeit, daß sich Werte auf der Oberfläche der bürgerlichen Gesellschaft nicht als solche darstellen, sondern notwendig die Form des (zufälligen) Marktpreises annehmen. Die bürgerliche Statistik drückt daher auch nur Aggregate des Gesamtprozesses aus, die in Marktpreisen bewertet sind. Hier geht es nun darum, sowohl für die Profitrate als auch für ihre bestimmenden Komponenten Indikatoren in der bürgerlichen Statistik aufzufinden, die die jeweiligen Faktoren und ihre Veränderung in der Zeit sichtbar machen, ohne diesen exakt entsprechen zu müssen.

Im folgenden werden wir daher versuchen, in der bürgerlichen Statistik den Marxschen Begriffen analoge Kategorien aufzufinden, und zwar für die

- Mehrwertrate, Mehrwertmasse,

-. organische Zusammensetzung,

- Profitrate,

- Akkumulationsrate, Wachstumsrate des Kapitals.

(Die analogen Begriffe für die ,organische Zusammensetzung“ des Kapitals werden erst unter 1.3 behandelt!)

Um die Entwicklung der Mehrwertmasse darstellen zu können, muß nun vom Nettoprodukt der Anteil abgezogen werden, der den Lohnarbeitern als Konsumtionsfonds zufällt. Hier ergibt sich nun eine weitere Schwierigkeit, die wir später in einem Exkurs andeutungsweise lösen werden: $\mathrm{C}$ a sowohl produktive wie unproduktive Arbeiter in der bürgerlichen Statistik begriffslos zusammengefaßt werden, können wir nur äußerst ungenau die Masse der produktiven, mehrwertsetzenden Arbeiter bestimmen. Wir werden dies später durch die Darstellung der Tendenzen im Verhältnis der verschiedenen Sektoren der Volkswirtschaft zueinander zu leisten versuchen, müssen jedoch uns hier auf die Verwendung der Lohnquote beschränken, wobei für uns auch hier nicht die absoluten Zahlen, sondern die Veränderung dieser Zahlen im Zeitablauf wichtig sind. Setzen wir also für das Nettoprodukt $Y$, die Lohnquote $w={ }_{Y}^{W} W=$ Lohnsumme, so läßt sich die Mehrwertmasse wie folgt darstellen:

$$
\text { (2) } \mathrm{m}=\frac{\mathrm{m}}{\mathrm{v}} \cdot \mathrm{v}
$$

(2') $\quad P=Y(1-w)$

wobei $P=$ Profitmasse

oder: $\mathrm{P}=\mathrm{Y}-\mathrm{W}$

Wir können, um die Mehrwertrate $\left(\frac{m}{V}\right)$ in bürgerlicher Schreibweise darzustellen, nur:mehr die Gleichung $\left(2^{\prime}\right)$ die Profitquote $\frac{P}{Y}$ wie folgt schreiben: $\frac{P}{Y}=\frac{\frac{P}{W} \cdot W}{Y}$ und erhalten so die umgeformte Lohnquote: $\frac{W}{Y}=1-\frac{\frac{P}{W} \cdot W}{Y}$ ! Die Mehrwertrate $\frac{\mathrm{P}}{\mathrm{W}}$ kann also - löst man die Gleichung für die Lohnquote nach $\frac{\mathrm{P}}{\mathrm{W}}$ auf, folgendermałsen geschrieben werden: (3) $\mathrm{m}^{\prime}=\frac{\mathrm{m}}{\mathrm{V}} \quad\left(3^{\prime}\right) \frac{\mathrm{P}}{\mathrm{W}}=\frac{\mathrm{Y}}{\mathrm{W}}-1$

Der Nenner der Profitrate wird in der bürgerlichen Statistik lediglich durch die Entwicklung des Kapitalstocks beschrieben. Damit fällt von vornherein der gesamte zirkulierende Teil des vorzuschießenden Kapitals $\left(=\mathrm{c}_{\mathrm{zirk}}+\mathrm{v}\right)$ aus der Betrachtung 
heraus. Wir werden dennoch mit dem Kapitalstock als Indikator für die Entwicklung des Nenners der Profitrate arbeiten und erst bei Untersuchung der Ergebnisse die notwendige Erweiterung des Nenners berücksichtigen. Demnach kann die (nunmehr auf die amtliche Statistik bezogene) Profitrate wie folgt beschrieben werden:

(4) $\mathrm{p}^{\prime}=\frac{\mathrm{m}}{\mathrm{v}} \cdot \frac{\mathrm{v}}{\mathrm{c}+\mathrm{v}}$;

Da $\frac{Y}{K}$
(4') $\pi=\frac{(1-W)}{K}$

wobei $\mathrm{K}$ gleich dem Kapitalstock.

mie ist, kann $\pi$ auch geschrieben werden als Produkt von Kápitalproduktivität und Profitquote $(1-W)$, die beide direkt in neueren statistischen Daten auffindbar sind. Die Größe $\pi$ wird auch als ,Kapitalrentabilität" bezeichnet.

(Eine weitere Einschränkung dieser hier für die bürgerliche Statistik umge. formten Formeln zur Profitrate und zur Mehrwertrate ist insofern notwendig, als darin nicht die Umschlagshäufigkeit des vorgeschossenen Kapitals zum Ausdruck kommt. Auch diesen Einwand können wir hier nicht weiter berücksichtigen, wenngleich gerade die Umschlagszahl erheblichen Einfluß auf Jahresmehrwertrate und

\section{Materialien und Untersuchungen - Ruhrgebiet}

Harry W. Jablonowski. Johanna Offermanns

\section{Teilkrise ohne Ende}

Eine Untersuchung über den industriellen "Strukturwandel" im Ruhrgebiet Welche Kapitalbewegungen stecken hinter der sogenannten "Strukturkrise“ im größten industriellen Ballungszentrum Europas? Mit welchen Strategien antworten Kapital und Staat auf die verschlechterten Verwertungsbedingungen in einzelnen Wirtschaftssektoren und Regionen (Zentralisation, Rationalisierungen, Entlassungen, Kapitalexport usw.)? Welche Konsequenzen hat diese Teilkrise für die Arbeiterklasse?

Unter diesen Fragestellungen werden zahlreiche Informationen aus Prognosen, Statistiken und Berichten wirtschaftswissenschaftlicher Forschungsinstitute usw. aufgearbeitet und gleichzeitig einige wichtige Untersuchungsmethoden der bürgerlichen Regionalanalyse kritisiert.

Die Arbeit kann also

1. über Methodenkritik hinaus Argumente gegen die Theorie und Propaganda von der Krisenelastizität des kapitalistischen Systems liefern;

2. methodische Anregungen für die Erstellung politökonomischer Analysen geben;

3. zur materialistischen Einschätzung der zahlreichen Teilkrisen in Industriezentren der EG, Japans und der USA beitragen, deren Charakter der bürgerliche Begriff "Strukturkrise" verdeckt.

Das Buch erscheint im Verlag des Politladen Erlangen Ende Juni 1974. Ca. 340 Seiten, ca. 13,00 DM. 
Profitrate haben durfte, wie auch aus der folgenden Formel ersichtlich, in der die Umschlagszahl in die Formel zur Bestimmung der Profitrate eingebaut ist (vgl. auch MEW 25, S. 84):

$$
\mathrm{p}^{\prime}=\min ^{\prime} \frac{\mathrm{v}}{\mathrm{c}+\mathrm{v}}
$$

(wobei n die Zahl der Umschläge des variablen Kapitals!))

Die Kapitalverwertung und damit die Profitrate als Motor der Produktion von Werten im Kapitalismus ist nun zwar Voraussetzung des Wachstums des Kapitals, aber nicht dessen hinreichende Bedingung. Wenn wir daher die Wachstumsrate des Kapitals (bzw. des Kapitalstocks) näher bestimmen wollen, müssen wir den akkumulationsfähigen Mehrwert resp. Profit näher bestimmen. Die Akkumulationsrate, das heißt der Teil des Mehrwerts, der wieder im Produktionsprozeß angelegt wird, kann nach Marx bestimmt werden als:

$$
\text { (5) } a=\frac{\Delta c+\Delta v}{M}
$$

d. h. der wieder im Produktionsprozeß anzulegende Teil des Mehrwerts muß sich aufspalten in zusätzliche Anlage von konstantem und zusätzliche Anlage von variablem Kapital. In der bürgerlichen Statistik könnte, unter der Voraussetzung, daß Sparen gleich Investieren ist (was durchaus nicht der Fall sein muß!), die Sparquote des Sparens aus dem Profit als Rate der Akkumulation einen Indikator für diese darstellen. Wird die Sparquote als $\underset{\mathrm{P}}{\mathrm{S}}$ bestimmt, so kann umgeformt die Gleichung (5) geschrieben werden: (17)

$$
\left(5^{\prime}\right) \quad a=\frac{S}{Y(1-w)}
$$

Nach diesen Überlegungen können wir nunmehr die Wachstumsrate des Kapitals bestimmen:

$$
\mathrm{ap}^{\prime}=\frac{\mathrm{am}}{\mathrm{v}} \cdot \frac{\mathrm{v}}{\mathrm{c}+\mathrm{v}} \quad\left(6^{\prime}\right) \quad \mathrm{a} \pi=\frac{\mathrm{a} Y(1-\mathrm{w})}{\mathrm{K}}
$$

Diese Wachstumsrate ist somit abhängig von der Entwicklung der Akkumulationsrate, des Nettoprodukts, der Lohnquote und der Größe des vorhandenen Kapitalstocks: mit dieser Wachstumsrate des Kapitals sind damit auch Veränderungen sowohl der technischen Durchschnittsbedingungen, des Verhältnisses von $\mathrm{G}$ und v, als auch der Wachstumsrate der wertbildenden Potenz, des Arbeitsvolumens, als auch der Angebots- und Nachfrageverhältnisse verbunden.

Insgesamt gilt für die Oberfläche der Gesellschaft als Ganzes und für die einzelnen Produktionssphären, daß sich Veränderungen der Profitrate nur in der statistisch erfaßten Form der Veränderung der Kapitalrentabilität (wie in $4^{\prime}$ ) entwickelt) darstellen. Eine solche Veränderung hat Konsequenzen für die Rate des Kapitalwachstums (wie aus $6^{\prime}$ ersichtlich); andererseits kann diese Veränderung aber

17) Die Akkumulationsrate ist nicht der in der bürgerlichen Statistik verfügbaren Investitionsquote gleich, diese mühte vielmehr so geschrieben werden:

$$
\mathrm{Iqu}=\frac{\mathrm{a} Y(1-\underline{w})}{\mathrm{y}}
$$


auch durch die gegenläufige Veränderung der Akkumulationsrate kompensiert werden.

\subsection{Zum Verhältnis von Profitrate und Kapitalrentabilität}

Wir wollen diesen letztgenannten Zusammenhang von Profitrate und Kapitalrentabilität näher ausführen und dabei auch versuchen, der organischen Zusammensetzung des Kapitals analoge Ausdrücke in der bürgerlichen Ókonomie in unsere Überlegungen einzuführen. Zum Zweck der Illustration sollen auch einige Resultate unserer empirischen Untersuchung hier vorweggenommen werden.

Mit Hilfe der Kapitalrentabilität können wir natürlich nur Trends in der Ver. änderung der Profitrate darstellen. Es geht uns dabei darum, die Beziehungen zu entwickeln, die zwischen der Profitrate p und der Kapitalrentabrität $\pi$ bestehen, die sich in dem statistischen Material ausdrückt. Da p' nicht mit $\pi$ identisch ist, können wir aus der Veränderung von $\pi$ nur Rückschlüsse auf die Wertbewegung ziehen.

Wir nehmen im folgenden die schon entwickelte Relation auf, in der sich die Bewegung der Profitrate reflektiert. Entsprechend der bügerlichen Theorie der Produktionsfaktoren wird in der Statistik die Kapitalproduktivität gemessen als Rela. tion von Nettoproduktionsvolumen zum Bruttoanlagevermögen. Das Bruttoanlagevermögen ist keineswegs mit dem konstanten Kapital identisch. Es ist erstens zu

Materialien und Untersuchungen - Ruhrgebiet

\section{Ruhrstahl und Imperialismus}

Analysen und Materialien zu Stahlkonzernen im Ruhrgebiet

Dieser Band enthält Untersuchungen über die fünf größten Stahlkonzerne der BRD.

Thyssen, Rheinstahl, Hoesch, Krupp und Mannesmann liegen sämtlich im Rhein-Ruhr-Gebiet und sind, wie bereits bis zum Ende des Faschismus, vielfältig miteinander verflochten. Die Stahlbranche ist ein entscheidender Faktor im BRD-Staat, der wachsende Einfluß des westdeutschen Stahlkapitals in der Europäischen Gemeinschaft wird in den Untersuchungem beispielhaft verdeutlicht.

Untersucht werden die Rationalisierungsinvestitionen, Verlagerung der Standorte zu den Küsten, Kapitalexport in die unterentwickelt gehaltenen Länder. Am Beispiel der Montan-Mitbestimmung wird gezeigt, daß Mitbestimmung und Gewerkschaftsapparat unter dem demokratischen Mäntelchen die ver. stärkte Ausbeutung der Arbeiterklasse absichern.

Das Buch erscheint im Verlag des Politladen Erlangen Ende September 74." Ca. 300 Seiten, ca. 14,00 DM. 
Markipreisen bewertet, zweitens umfaßt es nicht - wie schon gezeigt - den zirku* lierenden Teil des konstanten Kapitals, sondern nur den fixen Teil. Das Nettoproduktionsvolumen ist auch nicht identisch mit der Summe von variablen Teil des Kapitals und Mehrwert, es enthält außerdem die ,Abschreibungen", d, h. den verbrauchten Teil des konstanten fixen Kapitals. Die so problematisierte Kennziffer der Kapitalproduktivität wird nun multipliziert mit dem Profitanteil am Nettoproduktionsvolumen. Da dieser aber als solcher statistisch nicht ausgewiesen wird, muß man hier Hilfsgrößen hinzuziehen, nämlich den Anteil der Lohnkosten am Nettoproduktionswert (Lohnstiickkosten), der vom Gesamtproduktionswert subtrahiert wird. Natürlich ist dieses Vorgehen problematisch, da hier weder nach produktiver oder unproduktiver Arbeit, noch nach dem variablen Kapital als Kapitalvorschuß und den als Lohn bzw. Gehalt bezogenen Einkommen unterschieden werden kann. Die Formel, die wir oben entwickelt haben und hier verwenden werden, kann daher nur die Bewegung der Profitrate illustrieren:

$$
\pi=\frac{\mathrm{Y}}{\mathrm{K}}(1-\mathrm{W})
$$

Wenn man nun die so definierte Profitrate berechnet, ergeben sich für die 60er Jahre in der BRD folgende Ergebnisse, die wir im Vorgriff auf die genauere Darstellung im Text bereits hier darstelien:

\begin{tabular}{ccccc} 
Jahr & $\frac{\mathrm{Y}}{\mathrm{K}}$ & $\mathrm{w}$ & $1-\mathrm{w}$ & $\pi$ \\
\hline 1960 & 0,312 & 0,333 & 0,667 & 0,5416 \\
1961 & 0,791 & 0,355 & 0,645 & 0,5102 \\
1962 & 0,757 & 0,378 & 0,622 & 0,4709 \\
1963 & 0,727 & 0,389 & 0,611 & 0,4442 \\
1964 & 0,746 & 0,391 & 0,609 & 0,4543 \\
1965 & 0,741 & 0,413 & 0,587 & 0,4350 \\
1966 & 0,706 & 0,433 & 0,567 & 0,4003 \\
1967 & 0,653 & 0,430 & 0,570 & 0,3722 \\
1968 & 0,700 & 0,418 & 0,582 & 0,4074 \\
1969 & 0,754 & 0,428 & 0,572 & 0,4313 \\
1970 & 0,755 & 0,483 & 0,517 & 0,3903 \\
1971 & 0,729 & 0,520 & 0,480 & 0,3499 \\
\hline
\end{tabular}

Quelle: Eigene Berechnungen, Daten vom DIW, Produktionsvolumen und -potential, Produktionsfaktoren der Industrie im Gebiet der Bundesrepublik Deutschland, Statistische Kennziffern, 12. Folge, Berlin, September 1972

Es geht aus der Tabelle eindeutig der tendenzielle Fall der "Profitrate" $\pi$ der westdeutschen Industrie in den 60er Jahren hervor. Wir wollen an dieser Stelle nicht auf die darin eingeschlossenen Probleme hinsichtlich der Entwicklungsbedingungen des westdeutschen Kapitalismus eingehen; dies ist den ausführlichen Ausführungen unten vorbehalten. Hier geht es lediglich um die methodische Dimension des Problems. Die „Profitrate“ $\pi$, wie sie in der Tabelle dokumentiert ist, ist nicht identisch mit der Profitrate $\mathrm{p}^{\prime}$, wie sie Marx bestimmt hat, sondern ist die Kapitalrentabilität. In ihrer Veränderung aber dürfte sie ungefähr die Veränderung der Profitrate ange- 
ben. Denn wenn $p^{\prime}$ und $\pi$ auch ungleich sind, so besteht zwischen beiden doch eine Beziehung, die sich als Parallelität der Bewegung ausdriickt. Schon deshalb ist es möglich, $\pi$ als Indikator und Illustration fur die Veränderung der Profitrate $p^{\prime}$ zu verwenden und Schlußfolgerungen hinsichtlich der Akkumulationsbedingungen des Kapitals und der Kapitalanlage zu ziehen.

Einen höheren Aussagewert dieses Ergebnisses unserer empirischen Untersuchungen erhalten wir jedoch, wenn wir die Faktoren der Kapitalrentabilität weiter aufschlüseln. Marx leitet die historische Tendenz der Profitrate im wesentlichen aus der Verändermng der organischen Zusammensetzung des Kapitals ab. Daher müssen wir noch einen Indikator für die Veränderung der technischen und der wertmäßigen Zusammensetzung des Kapitals im Zeitablauf finden und diesen Indikator für die

\section{Materialien und Untersuchungen - Ruhrgebiet}

\section{Steinkohlenkrise im Ruhrgebiet und der Konkurrenzkampf auf dem Weltenergiemarkt}

Die Untersuchung geht von zwei Punkten aus: Zunächst wird die wirtschaftliche Entwicklung in der BRD nach dem 2. Weltkrieg in ihrer Abhängigkeit vom aufsteigenden US-imperialismus und ihre Beziehung zu den anderen europäischen Staaten dargestellt. Die Lage auf dem europäischen Energiemarkt wird in diesem Zusammenhang beleuchtet. Der zweite Ansatz geht von den Entwicklungen auf dem Weltenergiemarkt aus. Die beschleunigt expandierende Erdölproduktion der US-Konzerne im Nahen Osten Anfang bis Mitte der 50er Jahre bereitet unaufhaltsam die Überproduktionskrise am Ende jenes Jahrzehnts vor. Der faschistische Putsch im Iran 1953 und der SuezKrieg 1956 gegen Ägypten sind weltpolitische Schlaglichter dieser Entwicklung. Neben dem Erdöl wuchs die US-Kohle zu einem scharfen Konkurrenten der Ruhrkohle heran.

Überproduktion und sinkende ausländische Energiepreise stürzten die deutsche Steinkohle in eine immer noch andauernde Krise, in deren Folge 300000 Arbeitsplätze in anderthalb Jahrzehnten im Steinkohlenbergbau zerstört wurden. Aufgrund von Branchen- und Kapitalverflechtungen wurde das Ruhrgebiet davon besonders betroffen.

In dieser Analyse sollen auch die begrifflichen Konfusionen über die "Strukturkrise" im Kapitalismus grundlegend kritisiert und das Wesen der "Steinkohienkrise" eingeschätzt werden.

Die "Infrastruktur"-Aufgaben des Staates im Ruhrgebiet werden untersucht. Die Politik der Gewerkschaften wird im Verhältnis zum Staat und zu den Kämpfen der Kumpel gegen verschärfte Arbeitsintensität, Zechenstillegungen und Massenentlassungen zu beurteilen sein.

Das Buch erscheint im Verlag des Politladen Erlangen im Herbst 1974. Ca. 300 Seiten, ca. 14,00 DM. 
Formel der Kapitalrentabilität anwendbar machen. Als Indikator für eine steigende Zusammenseizung des Kapitals kann die Kapitalintensivierung genommen werden, die normalerweise durch den Ouotienten von Bruttoanlagevermögen und eingesetz. ten Arbeitsstunden ausgedrückt wird. Die Kapitalintensität ist also $\frac{K_{L}}{\mathcal{L}}$, wobei $\mathrm{K}$ das Bruttoanlagevermögen und $L$ die Anzahl der eingesetzien Arbeitsstunden bedeutet.

Andererseirs hat man aber noch aus den empirisch-statistischen Daten den Trend der Axbeitsprodzktivität zur Verfugung. Dieser ergibt sich aus der Entwicklung des Verhätnisses von Netroproduktionsvolumen zu eingesetzten Abeirssim. den $\left(\frac{Y}{L}\right)$. Die Kapitalintensität und die Arbeitsproduktivitat sind in der Statistik sowohl für jeden Indusiriezweig als auch für das verarbeitende Gewerbe und die Gesamtwirtschaft errechnet. Die Kapitalproduktivität in unserer Formel läßt sich aber nun mit Hilfe der Kapitalintensiäat und Abeitsproduktivität ausdrucken; die Kapitalprodukivitat ist nichts anderes als der Quotient dieser beiden Größen. Es ist also

$$
\frac{\mathrm{Y}}{\mathrm{K}}=\frac{\frac{\mathrm{Y}}{\mathrm{L}}}{\frac{\mathrm{K}}{\mathrm{L}}} \quad\left(=\frac{\text { Arbeitsproduknivicät }}{\text { Kapitalintersitat }}\right)
$$

Laßßt sich die Profitrate p' auf Basis der Wertheorie mit Hilfe der Mehrwertrate und der organischen Zusammensetzung ausdrücken, so lakst sich nun andererseits die Tendenz der Kapitalrentabiliät $\pi$ mit Hilfe

a) der Arbeitsproduktivität,

b) der Kapitalintensität

c) der Lohnquote darstellen.

Die beiden Profitraten $p^{\prime}$ und $\pi$ lassen sich nun unter Bezugnahme auf die organische Znsammensetzung in folgender Weise wieder gegenüberstellen:

$$
\text { (7) } \mathrm{p}^{\prime}=\frac{\mathrm{n}^{\prime}}{1+\frac{\mathrm{c}}{\mathrm{v}}}
$$$$
\pi=\frac{\frac{Y}{L}(1-w)}{\frac{K}{L}}
$$

Mî́ Hilfe der Zerlegung der Kapitalproduktivität als Quotient von Arbeitsproduktivität und Kapitalintensität sind aber damit auch bereits die Faktoren fü den Entwicklungstrend der Kapitalproduk tivität erklärt. Der in der ursprïnglichen Formel der Profitrate p' ausgedrückte einfache Sachverhalt - daß nämlich die Profitrate fällt, wenn der Kapitalstock schneller als das Nettoproduktionsvolumen steigt und die Einkommensverteilung konstant bleibt - läßt sich jetzt auch kausal in terpretieren. Nach der Formel ( $\left.7^{\prime}\right)$ sinkt die Profitrate dann, wenn mit Hilfe einer steigenden Kapitalintensivierung die Arbeitsproduk tivităt gesteigert werden soll, die Arbeitsproduktivität jedoch nur langsamer steigt als die Kapitalintensität und die Lohnquote konstant bleibt. Eine steigende, gleichbleibende oder fallende Kapitalrentabilität $\pi$-drei relevante Fälle für die historische Entwicklung in der BRD seit 1950 ! - erhält man also in folgender Weise: 
1. Die Profitrate $n$ steigt, wenn:

a) - die Arbeitsproduktivitä -- schneller steigt als

b) - - die Kapitalintevistat - und

c) - die Lohnquote - konstant bleibt oder womögich noch zusäzlich sinkt.

Dieser Fall gilt für die BRD von 1950 bis Ende der 50 er Jahre, als die Arbeitsproduktivitat stell anstieg (steigende Ausnutzung des fixen Kapitals etc.) und die Kapitalintensitä vergleichswise nur gering anstieg. Folghich stieg-wie spater noch näher ausgetuhrt wird -- die Kapitalproduktivitat (18); dariberhinaus sank noch die Iohnquote resp. dio Mehrwertiate stieg.

2. Die Profitrate or bleibt konstant, wenn:

a) - die Arbeitsproduktivitä -- ebenso schnell steigt wic

b) - die Kapitalintensität - und

c) - die Lonnquote - konstant bleibt oder deren Veränderung dirch eine gegenläufige Verändeng der Kapitalpodukrivität kompensientwird.

Dieser Fall gilt in der BRD Ende der $50 \mathrm{er} / \mathrm{Anfang}$ der $60 \mathrm{er}$ Jahre; nicht aber mehr für die Zet bis $1966 / 67$, da in diesem Zeitraun die Arbeitsproduktivität leicht absinkt und die Lohnquote leicht ansteigt (im Vergleich mit der vorherigen Periode) $(19)$.

18) Vgl dazu auch Whined Vogt, Die Wachstumszykler der westdeutschen Wirtschaft, Tübingen 1968

19) Vgl. dazu RFyorschungsprojekt, Wirtschaftliche und soziale Aspekte des technischen Wandels in der Bundesrepublik Deutschland", Driter Band: K..H. Oppenänder u. a., Wirtschatuche Auswirkungen des techischen Wandels in der Industrie, Frankturt a. M. 1971, S. 154

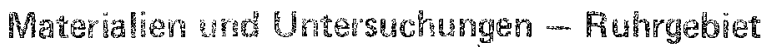

\section{Opel streikt!}

AnläBlich des Streiks bei Opel Bochura 1973 und der anschliaßenden Arbeits. gerichtsprozesse entstand dieser Bericht über den Streikverlaur, die Profite Opels, die Gberproduktionskrise der Automobilbranche und die imperialistische Strategie des Konzems General Motors, sowie über SPD. Staat und Ge. werkschatren als anti-proletarischer Front.

Das wird exemplarisch belagt an der Zerschlagung des Streiks. Der Bericht entstand aus der praktischen Solidaritat mit den kämpfenden Opel-kollegen. Er zeigt die Entwicklungstendenz der Automobilindustrie aut und versucht, Erfahrungen für die kommenden politischen Kämpfe der Arbeiterklasse weiterzugeberi.

Diese überarbeitete und erweiterte 2. Auflage einer bisher im Selbstverlag erschienenen Broschüre whd auch die Arbeitsgerichtsprozesse behandeln, die zur Zeit der ersten Ausarbeitung noch nicht abgeschlossen waren.

Das Buch erscheint in Verlag des Politaden Erlangen im Herbst 74 . Ca. 250 Seiten, ca. 11,00 DH. 
3. Die Profitrate $\pi$ fallt, wenn:

a) -- die Arbeitsproduktivität nicht so schnell steigt wie

b) - die Kapitalintensität - und

c) - die Lohnquote - konstant bleibr oder noch zusätzlich steigt.

Dieser Fall gilt fur die BRD von Anfang der 60er Jahre bis Anfang der 70 er Jahre und dauert - soweit erkennbar - noch an. Denn in diesem Zeitraum steigt die Kapitalintensitat in fast allen Industriezweigen enorm an bei gleichbleibender -- oder gar sinkender - Wachstumsrate der Arbeitsproduktivität und gleichbleibender oder steigender Lohnquote.

Diese obige Darstellung der Kapitalrentabilität als Ausdruck der Profitrate in ihren inneren Tendenzen soll uns in zweiten Abschnitt als Indikator für die Kapitalverwertung in einzelnen Phasen des Kapitalismus in der BRD dienen.

\subsection{Wertbildung und Kapitalverwertung unter Weltmarktbedingungen}

Wertbildung und Kapitalreproduktion wurden bisher betrachtet, als ob sie nur im nationalen Rahmen vonstatten gingen; beide vollziehen sich aber im Weltmarktzusammenhang. Dies gilt insbesonders für die BRD: Mehr als $20 \%$ des produzierten Warenkapitals werden regelmäßig exportiert; gleichermaßen sind die für den inneren Markt produzierter Waren der ausländischen Konkurrenz, dem Weltmarkt, ausgesetzt. Daher müissen wir die zusätzlichen methodischen Probleme erörtem, die sich fur unsere Analyse aus diesem Welmarktzusammenhang ergeben. Entsprechend unserer bisherigen Darstellung beschränken wir uns hier auf die Diskussion der Mow mente von Wertbildungs und Kapitalverwertungsprozek, wie wir sie schon entwickelt haben. Wir wollen uns zuerst wieder dem Wertbildungsprozes zuwenden.

Wie der Wert einer Ware sich durch die gesamte in dieser enthatenen Arbeit bemißt, die $z u$ den herrschenden technischen Burchschnittsbedingungen verausgabt worden sein muß, so ist entsprechend der Prozeß der Wertbildung eines Landes durch die Arbeit bestimmt, die zu den jeweiligen technischen Durchschnittsbedingungen in den Produktionsprozeß eingeht. Diese insgesamt produzierte Wertmasse wiederum wird bereits unter ganz bestimmten Distributionsverhältnissen prodiziert, wie etwa dem Verhältnis der unbezahlten zur bezahlten Arbeit, der Profitmasse zur Lohnmasse, dem akkumulierten und konsumierten Teil des Profits. Wir haben somit zwei Fragen zu untersuchen:

1. Welche Momente können die gesellschaftlichen Durchschnitısbedingungen der Arbeit einer Nation und daruber vermittelt deren Wertprodukt unter Weltmarktbedingungen bestimmen?

2. Welche Momente bestimmen unter Weltmarktbedingungen das Teilungsverhältnis dieses gesamten Wertprodukts, in dem sich die zu gesellschaftlichen Durchschnittsbedingungen verausgabte Arbeit darstellt?

Wie wir noch im einzelnen darstellen werden, ist die Phase beschleunigter AkkumuIation des westdeutschen Kapitalismus bis zum Anfang der 60er Jahre durch zwei Momente gekennzeichnet:

- durch raschen und beschleunigten Zustrom neuer Arbeitskraft in den kapita- 
listischen Produktionsprozeß auf der Grundlage weitgehend unveränderter technischer Bedingungen der Arbeit;

- durch eine im Vergleich zu den übrigen Industrienationen hohe Ausbeutungsrate, die nicht in einer außergewöhnlich hohen Produktivkraft der Arbeit ihre Ursache hatte.

Sobald die gesellschaftiche Durchschnittsbedingungen zu denen in den jeweiligen Branchen gearbeitet wird, mit anderen, überlegeneren Produk tionsbedingungen in Vergleich gesetzt werden, die die Arbeiter, die ihre Arbeitskraft zu diesen überlegenen Bedingungen verausgaben, in die Lage versetzen, dieselben Waren massenhaft zu niedrigeren Reproduktionskosten herzustellen, verändern sich die Voraussetzungen des (nunmehr unterlegenen) nationalen Wertbildungsprozesses. Der Wert derselben Waren bestimmt sich dort nun nicht mehr durch die bislang erforderlichen Arbeitsquanta, sondern durch diejenigen, die entsprechend den iberlegenen gesellschaftlichen Bedingungen notwendig sind: Wird die Arbeit in nationalem Rahmen nun nicht auch unter jenen international nunmehr vorherrschenden gesellschaftlichen Durchschnittsbedingungen verausgabt, zählt sie nicht mehr als ,Durchschnittsarbeit" und geht nicht mehr voll in den Wertbildungsprozeß ein.

Dieses Verhältnis zwischen wertbildender, gesellschaftlich notwendiger Arbeitszeit und massenhaft vorherrschenden technischen Bedingungen der Arbeit erklärt, weshalb die Wertbildung, die in den Industrienationen stattfindet, die zu unterlegenen Durchschnittsbedingungen produzieren, in zunehmendem Maße über den internationalen Zusammenhang vermittelt in ihrer extensiven Größe begrenzt̂ wird.

Das erreichte Ausmaß des internationalen Zusammengangs, der seit dem zweiten Weltkrieg entstanden ist und der sich anhand der internationalisierten Produktion, dem Umfang der getätigten Direktinvestitionen, der Zunahme des Welthandels und der Außenhandeisbeiträge zum Sozialprodukt der jeweiligen Industrienationen etc. konkretisieren läßt, dürfte dazu geführt haben, daß die technischen Durchw schnittsbedingungen der Arbeit, die in den jewelligen Branchen international vorherrschen entsprechend auch das Wertprodukt dieser Industrienationen berühren. Für wenig entwickelte Länder oder für nieđrig entwickelte Industriezweige innerhalb der Industrienationen wirkt sich der Weltmark tzusammenhang etwa dahingehend aus, daß die Reproduktionskosten der auf den Markt geworfenen Waren nicht mehr durch die eigene enthaltene vergegenständlichte Arbeit, sondern durch die Reproduktionskosten der auf dem Weltmark $t$ als Konkurrenz auftretenen, zu besseren Durchschnittsbedingungen produzierten und massenhaft augebotenen Waren bestimmt werden. Es genügt allerdings schon, daß die bloke Möglichkeit der verbilligten Reproduktion der Waren besteht, um die Arbeit der zu niedrigerén Durchschnittsbedingungen produzierenden Arbeiter zu entwerten (20).

Die äußerst rasche Kapitalbildung in der BRD bis zum Beginn der 60er Jahre, die mit einem ständig wachsenden Außenhandelsbeitrag zum BSP und einem stei-

20) So mag der andalusische Bauer heute sein Tagwerk in gleicher Zeit ableisten wie vor sagen wir einmal 20 Jahren und wird dennoch feststellen müssen, daf diese Arbeit sich nicht mehr in denselben Wertquanta darstellt wic vor 20 Jahren (weil nämlich international neuc Durchschnittsbedingungen inzwischen sich durchgesetzt haben). 
genden Anteil am Welthandel einherging, wäre demzufolge auch darauf zurickzuführen, daß die technischen Bedingungen, zu denen die Arbeitskräfte in den Produktionsprozeß der BRD eingegliedert wurden, im internationalen Vergleich gut gewesen sein müssen oder zumindest der Konkurrenz standgehalten haben. Für dieses Phänomen rasch anwachsender Masse verausgabter Arbeit bei unveränderter technischer Basis zumindest während der 50 er Jahre und zunehmender Bedeutung der BRD im Welthandel, muß jedoch noch ein weiteres zusätzliches Erklärungsmoment angeführt werden. Denn wie aus folgender Tabelle hervorgeht, waren (und sind) die Arbeitsproduktivitäten in den einzelnen Ländern (noch) sehr verschieden. Wenn dennoch die Länder mit unterdurchschnittlicher Arbeitsproduktivität z.T, überdurchschnittlich akkumulieren konnten, muß dies in einer hohen Ausbeutungsrate begründet sein.

Bruttowertschöpfung zu Fak torkosten je Beschäftigten 1962-1968 in der gesamten verarbeitenden Industrie

\begin{tabular}{l|c|c|c|c}
\hline & \multicolumn{2}{|c|}{ pro Jahr in Tausend Dollar } & \multicolumn{2}{c}{$\begin{array}{c}\text { v. H. } \\
\text { USA }=100\end{array}$} \\
\hline & 1962 & 1968 & 1962 & 1968 \\
\hline BRD & 3,1 & 4,8 & 35 & 41 \\
Frankreich & 3,8 & 6,1 & 43 & 52 \\
Italien & 1,9 & 3,2 & 22 & 27 \\
Niederlande & 2,9 & 5,3 & 33 & 45 \\
Belgien & 2,8 & 4,4 & 32 & 38 \\
EWG & 2,9 & 4,7 & 33 & 40 \\
Vereinigte Staaten & 8,8 & 11,7 & 100 & 100 \\
Japan & 1,7 & 3,3 & 9 & 28 \\
Vereinigtes Königreich & 2,7 & 3,4 & 31 & 29 \\
\hline
\end{tabular}

(Entnommen aus: Bericht über die Wettbewerbsfähigkeit der Europäischen Gemeinschaft, Band 1, (o. O.) Nov. 1971, S.2 III 20)

Es ließen sich weitere statistische Angaben anführen, die dieses Bild international unterschiedlicher Arbeitsproduktivität nach denjenigen Branchen aufgliedern, die in den Bereich der internationalisierten Produktion fallen, was jedoch hier zu weit führen würde. Wir dürfen jedoch von der unschwer belegbaren Annahme ausgehen, daß die Industrienation in den wichtigsten Weltmarktsbranchen (Chemie, Nahrungsmittel, Fahrzeug und Maschinenbau sowie Elektroindustrie) zu teilweise noch erheblich voneinander abweichenden nationalen Durchschnittsbedingungen der Arbeit produzieren (dabei können wir hier noch keine definitive Aussagen zu den technischen Durchschnittsbedingungen machen, da die internationalen Statistiken sich auf ökonomische Größen, nicht auf technologische Daten beziehen!). Im Fall des japanischen Kapitals, dessen Produktivkraft der Arbeit offensichtlich zumindest bis zum Ende der 60er Jahre unter dem Niveau der USA und auch unter demjenigen der westeuropäischen Kapitale lag, muß der bestehende in ternationale Zusammenhang, innerhalb dessen sich die japanische Exportproduktion bewegte, dazu geführt 
haben, daß die Arbeit, die in Japan in den Exportbranchen verausgabt wurde, nur zu Teilen wertbildend war, da die Durchschnittsbedingungen der Arbeit von den USA und von Westeuropa vorgegeben wurden. Die gleichzeitige beschleunigte Akkumulation des japanischen Kapitals ist deshalb vor allem durch die Exploitation der Arbeit des japanischen Arbeiters zu erklären. Extrem niedrige Reallönne ermöglichten es dem japanischen Kapital - trotz vergleichsweise geringer Produktivkraftentwicklung der Arbeit -. dennoch genügend akkumulierbare Mehrwertmassen zu produzieren, obwohl insgesamt die Wertbildung pro verausgabter Arbeitseinheit niedriger als in Ländern mit höherer Arbeitsproduk tivität sein mußte (21).

Dieser theoretische Zusammenhang, von verausgabter Arbeitszeit, deren Durchschnittsbedingungen weitgehend international vermittelt sind, Wertbildungsprozeß 3 und akkumulierbarer Mehrwertmasse, aufgrund extrem hoher Ausbeuiungsraten, wie im Fall des japanischen Kapitals, stellt also ein zusätzliches Erklärungsmoment auch für die relativ günstige Entwicklung des BRD-Kapitals während der 50 er Jahre dar

Die vergleichsweise rasche Kapitalbildung in der BRD, die sich bis zum Beginn der 60er Jahre auf der Grundlage einer nicht minder raschen Zunahme des BIP vollzog, kann ihre wesentlichen Voraussetzungen in folgenden zwei Momenten gehabt haben:

- die Durchschnittsbedingungen der Arbeit, zu denen die Arbeitskräfte in der BRD in den Produktionsprozeß eingegliedert worden sind, waren im in ternationalen Vergleich relativ gut und entsprachen in den in ternational konkurrierenden Branchen weitgehend den jeweiligen Durchschnittsbedingungen. Die in den betreffenden Branchen geleiste te Arbeitszeit konnte demzufolge in hohem Maße wertbildend wirken und jede Einstellung zusätzlicher Arbeiter (z. B. ausländische Arbeitskräfte) konnte entsprechend das Wertprodukt erhöhen.

- die Durchschnittsbedingungen der Arbeit, die in der BRD bis zum Ende 'rer 50er Jahre weitgehend unverändert fortbestanden haben, waren keineswegs im internationalen Vergleich (besonders nicht gegenüber den USA und Großbritannien) derart günstig, daß sich die rasche Zunahme des Sozialprodukts durch neu eingestellte Arbeiter und die beschleunigte Kapitalakkumulation ohne weiteres allein begründen ließen. Auf Basis der bisherigen Überlegungen ist vielmehr dann folgender Zusammenhang möglich: die niedrigen (Real-)Löhne hatten nicht nur eine hohe Mehrwertrate zum Ergebris, sondern drückten sich auch in (im internationalen Vergleich) niedrigen Kostpreisen der Waren

21) Dabei ist hervorzuheben, dats die Höhe der Ausbeutungsrate allein noch keinen Aufschluts über die Kompensationsmöglichkeiten für zurückgebliebene Produktivkraft auf dem Weltmarkt gibt. Vielmehr kommt es wesentlich auf die Bedingungen an, wodurch die Ausbeutungsrate hochgehalten wird: durch die Produktion des relativen Mehrwerts, d. h. durch eine hohe Produktivkraft der Arbeit mit der Konsequenz cines hohen Kapitalvorschusses oder durch Produktion des absoluten Mehrwerts, d. h. durch vergleichsweise niedrige Reallöhne, lange Arbeitszeiten and hohe Arbeitsintensität. Durch beide Methoden kann zwar die Ausbeutungstate gesteigert oder doch zumindest gehalten werden; aber nur die letztere Methode gewährleistet eine hohe Profitrate und bei disponibler Arbeitskraft auch besonders schnelle Akkumulationsraten! 
aus. Lag daher unter der Voraussetzung, daß die anderen Nationen zu besseren Durchschnittsbedingungen produzieren konnten, der Verkaufspreis der Waren auf dem Weltmarkt einerseits unter dem individuellen Wert der Waren (ein Teil der aufgewendeten Arbeit ging also nicht als wertbildend in den Produktionsprozeß ein), so konnte der Kapitalist dennoch aufgrund des niedrigen Kostpreises und der hohen Mehrwertrate einen angemessenen Durchschnittsprofit erzielen! (22)

Die Möglichkeit eines nationalen Kapitals, rascher zu akkumulieren als die Kapitale anderer Industrienationen, die zu besseren Durchschnittsbedingungen der $\mathbb{A}$ rbeit produzieren, hat also eine extrem hohe Ausbeutungsrate zur Voraussetzung, auf. grund derer das Kapital die relativ geringe Produktivität der Arbeit kompensieren und ausreichende Akkumulationsmasse erhalten kann. Diese Bedeutung des Teilungsverhältnisses des Neuwerts bzw. des Wertprodukts fur die Kapitalakkunulation gilt es noch weiter zu präzisieren, was wir durch einen kurzen Vorgriff auf die zweite Entwicklungsphase des Kapitals der BRD versuchen werden: Konnte das Kapital der BRD in den 50 er Jahren über weitgehend absolute Mehrwertproduktion bei steigendem Sozial- bzw. Wertprodukt rasch akkumulieren, so ist in den 60er Jahren, wie wir noch zeigen werden, dem westdeutschen Kapital dieser Weg der Akkumulation zunehmend versperrt; das Kapital ist unter diesen Bedingungen also die Produktivität der Arbeit anzuheben und durch die relative Mehrwertproduktion die Profitrate aufrechtzuerhalten und die für die Akkumulation benötigte Profitmasse zu produzieren (dies umso mehr, als die absolute Arbeitsmasse nunmehr begrenzt ist).

Nachdem wir bis hierher die Produktion der Warenwerte auf dem Weltmarkt unter dem Aspekt der Durchschnittsbedingungen betrachtet haben, können wir nun dazu übergehen, die Realisierungsbedingungen der produzierten Waren und die Vergleichung der bei der Produktion eingesetzten Kapitale (Profitrate) in unsere Uberlegıngen einzubeziehen.

Neben dem wechselseitigen Zusammenhang von Umfang des Wertprodukts (bzw. des Neuwerts), Verteilungsverhältnissen und Profitrate im kapitalistischen

22) Vgl. Karl Marx, Kapital Bd. 3, Berlin (DDR) 1964, S. 881, ,In der Konkurtenz sowohi der einzelnen Kapitalisten untereinander wie auf dem Weltmarkt sind es die gegebnen und vorausgesetzten Größen von Arbeitslohn, Zins, Rente, die in die Rechnung als konstante und regulierenden Größe eingehen; konstant nicht in dem Sinn, daß sie ihre Größen nicht ändern, sondern in dem Sinn, daß sie in jedem einzelnen Fall gegeben sind und đie konstante Grenze für die ständig schwankenden Marktpreise bilden. Z. B. bei der Konkurrenz auf dem Weltmarkt handelt es sich ausschließlich darum, ob mit dem gegebenen Arbeitslohn, Zins und Rente die Ware zu oder unter den gegebnen allgemeinen Marktpreisen mit Vorteil, d. h. mit Realisierung eines entsprecizenden Unternehmergewinns verkauft werden kann. Ist in einem Lande der Arbeitslohn und der Preis des Bodens niedrig, dagegen der Zins des Kapitals hoch, weil die kapitalistische Produktionsweise hier überhaupt nicht entwickelt ist, während in einem anderen Lande der Arbeitspreis und der Bodenpreis nominell hoch, dagegen der Zins des Kapitals niedrig steht, wendet der Kapitalist in dem einen Land mehr Arbeit und Boden, in dem andern verhältnismäßßig mehr Kapital an. In der Berechnung, wieweit hier die Konkurrenz zwischen beiden möglich, gehn diese Faktoren als bestimmende Elemente ein ... "“ 
Verwertungs- und Akkumulationsprozeß unter Weltmarktbedingungen ist nämlich noch ein weiteres Moment zu beachten, das sich aus der Notwendigkeit der Kontinuität des Reproduktionsprozesses selbst ergibt und das nicht minder eine Begrenzung für den Wertbildungsprozeß und Verwertungsprozeß darstellen kann. Die technischen Durchschnittsbedingungen der Arbeit, die jeweils in einer Branche gelten, und die bestimmen, in welchem Ausmaß die geleistete Arbeit überhaupt Wert konstituieren kann, unterliegen - wie bereits angedeutet - selbst wiederum einer beständigen Veränderung. Die Produktivkraftentwicklungen, die in den einzelnen Abteilungen und Branchen stattfinden, beeinflussen nicht nur den Wert der Ware Arbeitskraft (und dadurch die Mehrwertmasse), sondern vor allern auch die jeweils erforderliche proportionale Verteilung der gesamten verfügbaren gesellschaftlichen Arbeit über den Produktionsprozeß hinweg.

Diese proportionale Verteilung der gesellschaftlichen Arbeit auf die einzelnen Branchen setzt sich in der kapitalistischen Form des Reproduktionsprozesses krisenhaft durch, sei es durch Entwertungsprozesse, durch Brachlegung von produktiven Kapital, sei es durch partielle Überproduktion oder durch partielle Überakkumulation von Kapital in einzelnen Produktionszweigen, die alle die Freisetzung von Arbeitskräften zur Folge haben. So setzt sich das Wertgesetz in Krisen durch oder erzwingt durch teilweise Unterbrechung der Kontinuität der Reproduktion des Kapitals die jeweilige Verteilung der Arbeit und damit die Bedingungen der Wertbildung.

Die Realisierungsschwierigkeiten des Kapitals, in denen sich diese Prozesse niederschlagen, können demnach das Wertprodukt in seiner Größe reduzieren, obwohl sich die Produktion in den verschiedenen Industriezweigen zu den Durchschnittsbedingungen vollzieht. Der gesamtgesellschaftliche Reproduktionszusammenhang, in dem die Einzelkapitale stehen und in dem sie in einer stofflichen und wertmäßigen wechselseitigen Abhängigkeit miteinander verbunden sind, erfordert Kontinuität der Produktion. Sie ist eine Voraussetzung für die Reproduktion der Einzelkapitale. Diese skizzierte Bedeutung des Reproduktionszusammenhangs für die Realisierung und damit für die Größe des Wertprodukts wird insbesonders relevant, wenn der Weltmarktzusammenhang einbezogen wird. Die relativ große Außenhandelsverflechtung des BRD-Kapitals z. B. hat zur Folge, daß der Umfang der Reproduktion des westdeutschen Kapitals die Kontinuität der internationalen Zirkulation und das Fortbestehen des inernationalen Reproduktionszusammenhangs voraussetzt. Denn darüber ist die proportionale Verteilung der Arbeit beeinfluß und zur Vorbedingung der Produktion zahlreicher Branchen geworden. Der Umfang des Wertprodukts der BRD wie der des Wertprodukts jeden anderen Landes, dessen nationale Reproduktion in ähnlicher Weise den internationalen Zusammenhang einschließt, ist demnach nicht allein über die oben angedeuteten Zusammenhänge der Durchschnittsbedingungen der Arbeit im intemationalen Vergleich, sondern auch von der Möglichkeit der Realisierung auf dem Weltmarkt abhängig.

Wenn wir zuvor die Möglichkeiten eines nationalen Kapitals dargestellt haben, bei niedriger Produktivität dennoch Wertbildung, Realisierung und Akkumulation in der internationalen Konkurrenz zu gewährleisten (etwa durch eine hohe Exploitationsrate), so sind dies zugleich auch jene Momente, durch deren Veränderung die 
nationale Profitrate, Akkumulation und die Stellung auf dem Weltmarkt (internationale Konkurrenzsituation) negativ beeinflußt werden können.

Die Verschlechterung der Verwertung kann z. B. aufgrund von Reallohnsteigexungen, die Folge verschärfter Lohnkämpfe und/oder eines stagnierenden Arbeitsvolumens sind, eintreten; dies besonders dann, wenn die Zunahme der Arbeitsproduktivität (das Mittel der relativen Mehrwertproduktion) ebenfalls zurückbleibrt Die Verschlechterung der Verwertung kann aber auch dann eintreten, wenn die Produktivkraft der Arbeit mit wachsendem Kapitalaufwand und daher wachsender Kapitalintensität zu steigern versucht wird.

Schematisch läßt sich der hier entwickelte theoretische Zusammenhang zwischen Wertbildung und Kapitalreproduktion wie folgt zusammenfassen:

Die Durchschnittsbedingungen, (die einen internationalen Vergleichszusammenhang einschließen) zu denen die Masse der Arbeit verausgabt wird, bilden die Basis für die Größe des Wertprodukts (bzw. des Neuwerts). Dieses Wertprodukt wird bereits un. ter bestimmten Exploitationsverhältnissen erstellt, von denen die Profit- und Akkumulationsrate mit abhängen, die ihrerseits über die Konkurrenz vermittelt zur Steigerung der Produktivkraft führen und somit auf die techniscr zn Bedingungen der Arbeit, d. h, die Basis der Wertbildung zurückwirken, andererseits aber auch die Größe der in den Produktionsprozeß eingehender Arbeitsmasse bestimmen; beides bildet wiederum die Basis des Wertbildungsprozesses. Diese wechselseitigen Bedingungen von Wertprodukt und Profitrate hängen insgesamt von den stofflichen und wertmäßigen Proportionen im kapitalistischen Reproduktionsprozeß ab, der den internationalen Zusammenhang einschließt.

[Schluß 1. Teil, zweiter Teil in PROKLA 14/15]

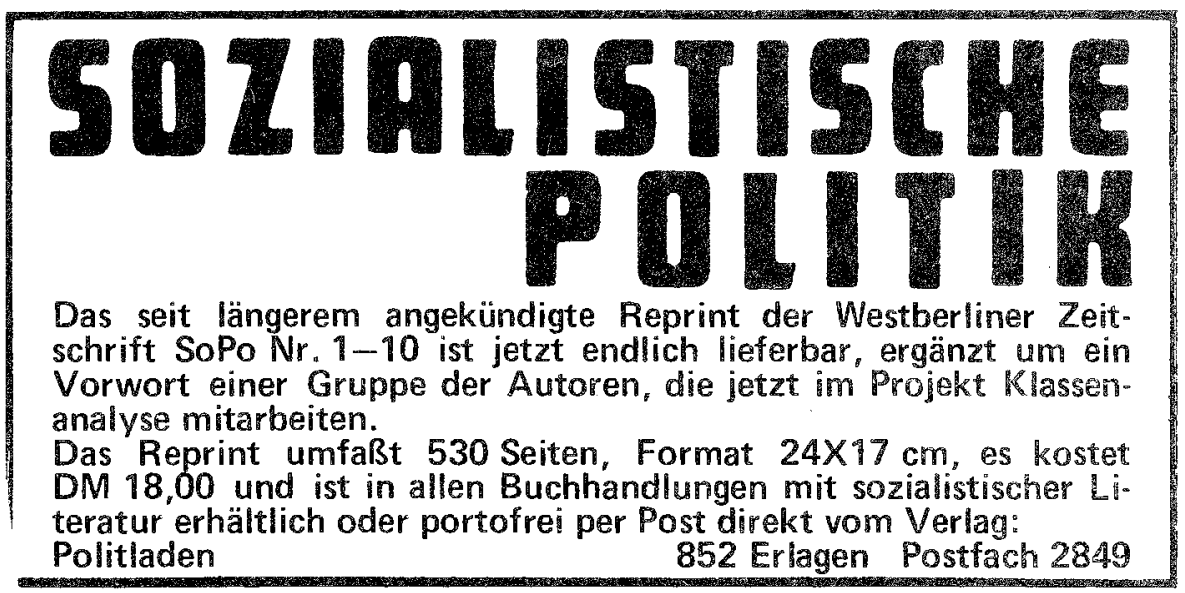

